\title{
Local preconditioning and variational multiscale stabilization for Euler compressible steady flow
}

\author{
Margarida Moragues Ginard ${ }^{\mathrm{a}, *}$, Mariano Vázquez ${ }^{\mathrm{a}, \mathrm{b}}$, Guillaume Houzeaux $^{\mathrm{a}}$ \\ ${ }^{a}$ Barcelona Supercomputing Center, Spain \\ ${ }^{b}$ IIIA-CSIC Barcelona, Spain
}

\begin{abstract}
This paper introduces a preconditioned variational multiscale stabilization (P-VMS) method for compressible flows. In this introductory paper we focus on inviscid flow and steady state problems. The Euler equations are solved on fully unstructured grids and discretized using the finite element method. The P-VMS method can be decomposed in three parts. First, a local preconditioner is applied to the continuous equations to reduce the stiffness while covering a wide range of Mach numbers. Then, the resulting preconditioned system is discretized in space using finite elements and stabilized with a variational multiscale stabilization method adapted for the preconditioned equations. In this paper, the solution is advanced in time using a fully explicit time discretization, although P-VMS is general and can be applied to fully implicit solvers. The proposed method is assessed by comparing convergence and accuracy of the solutions between the non-preconditioned and preconditioned cases, in particular for van Leer-Lee-Roe's [1] and Choi-Merkle's [2] preconditioners, in some selected examples covering a large range of Mach numbers.
\end{abstract}

Keywords: Local preconditioning, variational multiscale method, finite elements, Euler equations, compressible flow, steady flow problems.

\section{Introduction}

As it is well known, the characteristic propagation speeds of the Euler system of equations, $\{c M, c(M+1), c(M-1)\}$, being $c$ the speed of sound and $M$ the Mach number, lead to highly disparate values especially when the Mach number approaches 0 or 1 . This disparity is paired with a strong increase in the system's stiffness and a consequent loss in convergence speed. As this behavior precedes the discretization, it happens regardless of the numerical method that is used.

*margarida.moragues@bsc.es 
The goal of local preconditioning is the uniformization of the characteristic propagation speeds of the system. This entails a gain in the convergence speed to a steady state or to the transient solution at a given time step when a pseudo-time technique $[3,4]$ is used. Local preconditioning also gives accuracy to the solution as it is shown, for example, in [5] for finite difference methods. Local preconditioning is applied to the set of equations before any discretization is done. The extra computational cost is minimal. It consists of transforming the original convective jacobians of the Euler system into the preconditioned ones, in such a way that the non-preconditioned and the preconditioned equations have different time evolution but reach the same steady state.

Based on the idea of artificial compressibility of Chorin [6], local preconditioning is first set up by Turkel [7] for incompressible and low speed compressible flow. Other local preconditioners have been presented since then. Van Leer-Lee-Roe's (VLR) preconditioner $[1,8]$ is introduced by van Leer et al. for Euler steady flow covering a wide range of Mach numbers. It is extended to NavierStokes in [9, 10]. In [2], Choi-Merkle's (CM) preconditioner is presented for low Mach number steady flow, suitable for both the Euler and Navier-Stokes equations with a single parameter change. It is extended to transient flows in [11]. Weiss and Smith [12] propose a preconditioner to solve incompressible and variable density transient flows in transonic and low-speed regimes. The diagonal preconditioner defined by Briley et al. [13] is applicable to unsteady viscous flows, for Mach numbers ranging from essentially incompressible to supersonic. Local preconditioning is independent of the space discretization method; in all the references listed above the finite differences and finite volumes methods are used. In the context of the finite volume discretization, the Roe's flux is adapted to the preconditioned equations, for example, in $[14,15]$ for the VLR preconditioner. For the central difference scheme, the artificial viscosity should be based on the preconditioned equations as well [16]. As far as we know, there are two precedents in the use of finite elements (FE) and local preconditioners: [11] and [17], where the CM preconditioner is used for the solution of compressible viscous flow in steady and transient problems, respectively.

In this work, we introduce a FE space discretization of a locally preconditioned system, using the so-called variational multiscale stabilization (VMS) method. The VMS term of the original (non-preconditioned) equations is modified in order to stabilize the preconditioned system. We will show that due to both the way VMS is designed and how local preconditioning acts upon the hyperbolic set of equations, their combined effect leads to a robust and precise numerical 
method, which we call preconditioned VMS, or P-VMS. For the sake of simplicity, in this paper we present the method by focusing on the compressible Euler equations for steady state problems. Its performance is assessed on linear explicit schemes where global and local time-stepping are compared. Inviscid VLR and CM preconditioners are used and tested for low Mach, transonic, and supersonic regimes. A Fourier stability analysis of the P-VMS method for the Euler equations of steady flow, and some improvements, are presented in a companion paper [18]. Preliminar results of the P-VMS method applied to the Navier-Stokes equations and transient flow problems can be found in [19]. First results on the use of an implicit time scheme are obtained as well [20], showing great improvement in the convergence rate.

It is important to notice that local preconditioning comes before any discretization method is applied to the equations. Once discretized, the resulting algebraic system could eventually present disparities in its eigenvalues, related for example to different element sizes on the mesh, different values of velocity, density, etc. over the domain. Therefore, local preconditioning effects could even improve if additional algebraic preconditioners are applied as well. We will describe this strategy in a future paper, where the implicit P-VMS scheme is presented.

The paper is organized as follows. The Euler equations of compressible flows are presented in Section 2. The condition number of the Euler equations and the stiffness problem is discussed in Section 3. In Section 4 we introduce the preconditioned Euler equations. Two preconditioners, VLR and CM, are also introduced in this section and their condition numbers are discussed. The discrete formulation of the system and the P-VMS method are presented in Section 5. The improvement of local preconditioning in terms of rate of convergence and accuracy of the steady solution is shown through the examples of Section 6. The paper ends with a group of appendices where useful algebra is made explicit.

\section{Euler equations}

Given a bounded domain $\Omega \subset \mathbb{R}^{3}$, the three dimensional Euler equations for compressible flow can be written in the conservative form as follows:

$$
\frac{\partial \boldsymbol{\Phi}}{\partial t}+\frac{\partial \mathbf{F}^{i}(\boldsymbol{\Phi})}{\partial x_{i}}=0
$$


for $i=1, \ldots, 3$ which label the space dimension. We use the Einstein summation convention that implies summation over repeated indexes in the same term. In (1), $\mathbf{F}^{i}$ are the convective fluxes:

$$
\mathbf{F}^{1}=\left[\begin{array}{l}
\frac{U_{1} U_{1}}{\rho}+p \\
\frac{U_{2} U_{1}}{\rho} \\
\frac{U_{3} U_{1}}{\rho} \\
U_{1} \\
\frac{U_{1}}{\rho}(E+p)
\end{array}\right], \quad \mathbf{F}^{2}=\left[\begin{array}{l}
\frac{U_{1} U_{2}}{\rho} \\
\frac{U_{2} U_{2}}{\rho}+p \\
\frac{U_{3} U_{2}}{\rho} \\
U_{2} \\
\frac{U_{2}}{\rho}(E+p)
\end{array}\right], \quad \mathbf{F}^{3}=\left[\begin{array}{l}
\frac{U_{1} U_{3}}{\rho} \\
\frac{U_{2} U_{3}}{\rho} \\
\frac{U_{3} U_{3}}{\rho}+p \\
U_{3} \\
\frac{U_{3}}{\rho}(E+p)
\end{array}\right],
$$

and $\boldsymbol{\Phi}$ is the vector of the unknowns in conservative variables:

$$
\boldsymbol{\Phi}=\left(U_{1} U_{2} U_{3} \rho E\right)^{\mathrm{T}}
$$

where $U_{1}, U_{2}, U_{3}$ are the components of the momentum in the three space directions, $\rho$ is the density, and $E$ is the total energy per unit volume. All the unknowns are functions of space $\mathbf{x}=\left(x_{1}, x_{2}, x_{3}\right)$ and time $t$. We note $\mathbf{U}$ the momentum vector, that is $\mathbf{U}=\left(U_{1}, U_{2}, U_{3}\right)$. The superscript $\mathrm{T}$ represents the transposed vector. Other important physical variables are: the velocity $\mathbf{u}=\frac{\mathbf{U}}{\rho}$, the Mach number $M=\frac{\|\mathbf{u}\|}{c}$, the pressure $p=\frac{R}{c_{\mathrm{v}}}\left(E-\frac{1}{2} \frac{U_{k} U_{k}}{\rho}\right)$, the temperature $T=\frac{1}{c_{\mathrm{v}} \rho}\left(E-\frac{1}{2} \frac{U_{k} U_{k}}{\rho}\right)$, where $R=c_{\mathrm{p}}-c_{\mathrm{v}}$ is the constant of perfect gases, and $c_{\mathrm{p}}$ and $c_{\mathrm{v}}$ are the coefficients of specific heat at constant pressure and volume, respectively. In this paper $\|\cdot\|$ represents the $L^{2}$ norm.

Using the Jacobian matrices, $\mathbf{A}^{i}(\boldsymbol{\Phi})=\frac{\partial \mathbf{F}^{i}}{\partial \boldsymbol{\Phi}}$, whose explicit expression can be found in Appendix A, the three-dimensional Euler equations can be re-written in non-conservative form as follows:

$$
\frac{\partial \Phi}{\partial t}+\mathbf{A}^{i}(\boldsymbol{\Phi}) \frac{\partial \Phi}{\partial x_{i}}=0
$$

The problem is to find $\mathbf{\Phi}(\mathbf{x}, t)$ satisfying Eq. (4) with proper initial and boundary conditions, for all $(\mathbf{x}, t) \in \Omega \times \mathbb{R}^{+}$. The Euler equations (4) are hyperbolic equations because for any linear combination of the form $\mathbf{A}_{\mathbf{k}}=k_{i} \mathbf{A}^{i}$, with $k_{i} \in \mathbb{R}, \mathbf{A}_{\mathbf{k}}$ is diagonalizable with real eigenvalues. In this work, (4) is discretized in time using a first order forward finite difference scheme, leading to an explicit formulation. An explicit time integration is used to introduce the method for the sake of simplicity. However this is not the best choice in terms of convergence speed of the method. 


\section{Stiffness and condition number}

\subsection{One-dimensional case}

In order to fully understand the effect of local preconditioning, let us first analyze the onedimensional case. A system of the form

$$
\frac{\partial \boldsymbol{\Phi}}{\partial t}+\mathbf{A}(\boldsymbol{\Phi}) \frac{\partial \boldsymbol{\Phi}}{\partial x}=0
$$

is called hyperbolic if the matrix $\mathbf{A}$ is diagonalizable with real eigenvalues. These eigenvalues are called the characteristic propagation speeds of the system. It is widely known that marching in time an hyperbolic system containing different propagation speeds presents some problems. By the CFL condition [21] the time step should be $\Delta t \leq \frac{h}{|\lambda|_{\max }}$, where $h$ is a characteristic length of the grid and $|\lambda|_{\max }$ the largest absolute value of the eigenvalues of the matrix $\mathbf{A}$. A common time step for all the equations means that some modes would use a time step that is not the best not only in terms of accuracy but also in terms of efficiency; this is because the fast waves impose their small time steps to the slow ones. In the one dimensional case, the propagation speeds are the eigenvalues of the convective jacobian: $\lambda(\mathbf{A})=\{|u|-c,|u|+c,|u|\}=c\{M-1, M+1, M\}$. When $M \rightarrow 0$ or $M \rightarrow 1$ a large disparity occurs between the eigenvalues, resulting in a non efficient time marching of the equations.

Although this happens regardless of the time-space discretization that is utilized, its effect is blatantly observed in the discretized form, because any discretization of the system would be more robust if all the modes traveled at the same speed. A system of hyperbolic equations is said to be stiff or ill-conditioned when it exists a big disparity between its eigenvalues or characteristic speeds. In that case, convergence problems arise regardless of the discretization method that is used. The stiffness of a system is measured by the so-called condition number or characteristic condition number, defined in the following.

Let us recall that the condition number of a matrix $\mathbf{A}$ is defined as

$$
\kappa(\mathbf{A})=\frac{|\lambda(\mathbf{A})|_{\max }}{|\lambda(\mathbf{A})|_{\min }}
$$

where $|\lambda(\mathbf{A})|_{\max }$ and $|\lambda(\mathbf{A})|_{\min }$ are respectively the maximum and the minimum absolute values of the eigenvalues of $\mathbf{A}$. In one dimension, the characteristic condition number, $\kappa$, of the Euler system 
of equations (5) corresponds to the condition number of its jacobian matrix, $\kappa(\mathbf{A})$, that is

$$
\kappa= \begin{cases}\frac{M+1}{M} & M<\frac{1}{2} \\ \frac{M+1}{1-M} & \frac{1}{2} \leq M \leq 1 \\ \frac{M+1}{M-1} & 1<M .\end{cases}
$$

\subsection{Multi-dimensional case}

In the multi-dimensional case, if $d$ is the space dimension, there is one Jacobian $\mathbf{A}^{i}$ for each space direction $i=1, \ldots, d$. Therefore, the definition of the condition number is not as obvious as it is for the one dimensional case and wave analysis becomes a necessary mathematical tool to evaluate the stiffness of 2D and 3D hyperbolic equations as the Euler equations (4). Examples of its use can be found in $[8,10,1,22,14]$. The idea is to compute the condition number by analyzing the wave propagation (or wave speed) of plane waves. The basic rationale is the following. A plane wave of the form $\mathbf{\Phi}(\mathbf{n} \cdot \mathbf{x}-\lambda t)$ is plugged into (4), where $\mathbf{n}$ is a unit vector defining the direction of propagation and $\lambda$ is the propagation speed of the plane wave. Thus we find the eigenvalue problem

$$
\left(\mathbf{A}_{\mathbf{n}}-\lambda \mathbf{I}\right) \boldsymbol{\Phi}^{\prime}=0
$$

where $\mathbf{A}_{\mathbf{n}}=\sum_{i=1}^{d} \mathbf{A}^{i} n_{i}$ and $\mathbf{I}$ is the $d \times d$ identity matrix. Then, the wave speeds in the propagation direction $\mathbf{n}$ are the eigenvalues $\lambda_{j}\left(\mathbf{A}_{\mathbf{n}}\right), j=1, \ldots, d+2$.

As in the one-dimensional case, to respect the CFL condition the time step should be

$$
\Delta t \leq \frac{h}{|\lambda|_{\max }}
$$

where $h$ is a characteristic length of the grid and

$$
|\lambda|_{\max }=\max _{j, \mathbf{n}}\left|\lambda_{j}\left(\mathbf{A}_{\mathbf{n}}\right)\right|
$$

is the maximum absolute value of the eigenvalues of the problem. The characteristic propagation speeds of the Euler equations in the reference coordinates are:

$$
\begin{aligned}
& \lambda_{1, \ldots, d}=\mathbf{u} \cdot \mathbf{n} \\
& \lambda_{d+1, d+2}=\mathbf{u} \cdot \mathbf{n} \pm c .
\end{aligned}
$$


In streamline coordinates (in Appendix C) they write:

$$
\begin{aligned}
& \lambda_{1, \ldots, d}=c M n_{1} \\
& \lambda_{d+1, d+2}=c\left(M n_{1} \pm 1\right) .
\end{aligned}
$$

As we consider all the unit vectors $\mathbf{n}$, the ensembles (11) and (12) turn to be the same. In fact, the reference coordinates and the streamline coordinates always give the same ensemble of eigenvalues. The largest absolute value of the eigenvalues is

$$
|\lambda|_{\max }=c(M+1)
$$

defining the time steps for the marching scheme. The goal of preconditioning is therefore to cluster the eigenvalues (12) as much as possible.

The natural extension of expression (6) to the multi-dimensional case is

$$
\frac{\max _{i, \mathbf{n}}\left|\lambda_{i}\left(\mathbf{A}_{\mathbf{n}}\right)\right|}{\min _{i, \mathbf{n}}\left|\lambda_{i}\left(\mathbf{A}_{\mathbf{n}}\right)\right|}
$$

However this is not a good measure of the problem stiffness. This is because the values $\lambda_{1, \ldots, d}\left(\mathbf{A}_{\mathbf{n}}\right)$ are zero when $\mathbf{n}$ is orthogonal to the velocity $\mathbf{u}$, so the denominator $\min _{i, \mathbf{n}}\left|\lambda_{i}\left(\mathbf{A}_{\mathbf{n}}\right)\right|$ is always zero regardless of the local Mach number. Therefore, the group velocity is instead used in preconditioning analysis $[8,22]$ to define the condition number. The group velocity is the propagation speed of the envelope of a plane wave defined as

$$
\mathbf{g}_{i}(\mathbf{n})=\nabla_{\mathbf{n}}\left(\|\mathbf{n}\| \lambda_{i}(\mathbf{n})\right)
$$

For an equivalent way of computing the group velocity, refer to Appendix E. The condition number is defined as the ratio of the largest and the smallest magnitude of the group speeds

$$
\kappa=\frac{\max _{i, \mathbf{n}}\left\|\mathbf{g}_{i}(\mathbf{n})\right\|}{\min _{i, \mathbf{n}}\left\|\mathbf{g}_{i}(\mathbf{n})\right\|} .
$$

Regardless of the space dimension, by plugging (12) into (15) (or into (E.1)-(E.4) from Appendix E), the group velocities, $\mathbf{g}(\mathbf{n})$, for the Euler system (4) are

$$
\begin{aligned}
& \mathbf{g}_{1, \ldots, d}=(c M, 0, \ldots, 0)^{\mathrm{T}} \\
& \mathbf{g}_{d+1, d+2}=\left(M \pm n_{1}, n_{2}, \ldots, n_{d}\right)^{\mathrm{T}}
\end{aligned}
$$


and their magnitudes are

$$
\begin{aligned}
& \left\|\mathbf{g}_{1, \ldots, d}\right\|=c M \\
& \left\|\mathbf{g}_{d+1, d+2}\right\|=c \sqrt{M^{2}+1 \pm 2 M n_{1}} .
\end{aligned}
$$

Therefore the condition number of system (4) is

$$
\kappa= \begin{cases}\frac{M+1}{M} & M<\frac{1}{2} \\ \frac{M+1}{1-M} & \frac{1}{2} \leq M \leq 1 \\ \frac{M+1}{M-1} & 1<M .\end{cases}
$$

\section{Local preconditioning}

The preconditioned Euler system reads

$$
\frac{\partial \boldsymbol{\Phi}}{\partial t}+\mathbf{P A}^{i}(\boldsymbol{\Phi}) \frac{\partial \boldsymbol{\Phi}}{\partial x_{i}}=0,
$$

where $\mathbf{P}$ is the preconditioner. As noted in $[10,22]$, assuming that $\mathbf{P}$ has non-zero determinant and (20) converges to a steady state solution, the time derivatives go to zero and (4) and (20) will share the same steady solution. In this section, the VLR and CM preconditioners are described and briefly analyzed.

A preconditioner is said to be optimal if it reduces the stiffness of the system to the minimum attainable. Optimality is, in fact, the main goal of preconditioning. However, some essential considerations have to be taken into account [10, 22]:

- Positivity. The preconditioner should not reverse the direction of the propagation speeds of the system. Otherwise incoming and outgoing waves would inverse roles and cause problems for the imposed boundary conditions. This requirement is satisfied if $\mathbf{P}$ is a positive-definite matrix.

- Symmetrizability. In order to guarantee that the energy of the system remains bounded, the system of equations should remain symmetrizable after preconditioning. This means that it exists a symmetric, positive definite matrix $\mathbf{S}$, such that $\mathbf{S} \mathbf{P} \mathbf{A}^{i}$ is a symmetric matrix for all $i=1, \ldots, d$. 
- Continuity. The preconditioner should satisfy some continuity conditions through all Mach number regimes. This is especially sensitive at the sonic point (i.e.: when $M=1$ ) where a smooth transition between the subsonic and the supersonic preconditioners is required.

As explained in $[1,8]$, in the one-dimensional case, the optimal condition number we can obtain when preconditioning is 1 , in two dimensions it is 1 for supersonic flow and $\frac{1}{\sqrt{1-M^{2}}}$ for subsonic flow, and in three dimensions it is $\frac{1}{\sqrt{1-M^{2}}}$ for subsonic and $\frac{M}{\sqrt{M^{2}-1}}$ for supersonic flow. This is summarized in Table 1.

\begin{tabular}{l|c|c|c|c} 
& No precond. & \multicolumn{3}{|c}{ Optimal preco } \\
\cline { 3 - 5 } & & $1 \mathrm{D}$ & $2 \mathrm{D}$ & $3 \mathrm{D}$ \\
\hline Subsonic & $\frac{M+1}{\min \{M, 1-M\}}$ & 1 & $\frac{1}{\sqrt{1-M^{2}}}$ & $\frac{1}{\sqrt{1-M^{2}}}$ \\
Supersonic & $\frac{M+1}{M-1}$ & 1 & 1 & $\frac{M}{\sqrt{M^{2}-1}}$
\end{tabular}

Table 1: Condition number of the non-preconditioned Euler equations and optimal condition number of the preconditioned Euler equations.

We express and solve the Euler equations in conservative variables (3), which are used in our simulation code. However the preconditioners are designed and introduced through different variables sets. These sets are the so-called symmetrizing variables, symmetrizing variables with streamline coordinates, and primitive variables, which are described in Appendix B, Appendix C, and Appendix D, respectively.

Condition number. In the same way as it is done in Section 3 for the non-preconditioned Euler equations (4), wave analysis is used for the preconditioned case (20). The characteristic propagation speeds, group velocities, and condition number of (20) are defined in this paragraph, and its value for the VLR and CM preconditioners is computed in Subsections 4.1 and 4.2, respectively.

The characteristic propagation speeds or eigenvalues of system (20) are the propagation speeds of a plane wave solution of the system, which are the eigenvalues of $\mathbf{P} \mathbf{A}_{\mathbf{n}}=\sum_{i=1}^{d} \mathbf{P} \mathbf{A}^{i} n_{i}$, that is: $\lambda_{j}\left(\mathbf{P A}_{\mathbf{n}}\right), j=1, \ldots, d+2$, where $\mathbf{n}$ is a unit vector defining the direction of propagation of the plane wave. The largest eigenvalues absolute value of the preconditioned problem (20), defined by

$$
|\lambda|_{\max }=\max _{j, \mathbf{n}}\left|\lambda_{j}\left(\mathbf{P} \mathbf{A}_{\mathbf{n}}\right)\right|
$$


is the one expected to determine the time step for this problem. This subject is discussed in more detail in Subsection 5.3.

In an analogous fashion as it is defined in Subsection 3.2, the group velocities of the preconditioned Euler system (20) are defined by

$$
\mathbf{g}_{i}(\mathbf{n})=\nabla_{\mathbf{n}}\left(\|\mathbf{n}\| \lambda_{i}(\mathbf{n})\right)
$$

where $\left.\lambda_{i}(\mathbf{n})\right)=\lambda_{i}\left(\mathbf{P A}_{\mathbf{n}}\right)$, for $i=1, \ldots, d$. An equivalent way to compute the group velocities is found in Appendix E. The condition number is defined by Eq. (16) that we repeat here for the sake of clarity:

$$
\kappa=\frac{\max _{i, \mathbf{n}}\left\|\mathbf{g}_{i}(\mathbf{n})\right\|}{\min _{i, \mathbf{n}}\left\|\mathbf{g}_{i}(\mathbf{n})\right\|} .
$$

In (23), the group velocities of the preconditioned Euler system (22) are used.

The expressions of the eigenvalues, the maximum absolute value of the eigenvalues, and the condition number for the unpreconditioned system and for the VLR and CM preconditioned systems are summarized in Tables 2 and 3 for the two and three dimensional cases, respectively. These values do not depend on the set of variables that we chose to express our system of equations.

\subsection{VLR preconditioner}

The VLR preconditioner for the Euler equations is obtained by imposing a general preconditioning matrix that satisfies a number of properties such as: optimality, accuracy, continuity at the sonic point, preservation of the decoupled entropy equation, positivity, and symmetrizability. The VLR preconditioner is introduced in $[8,10]$ using the symmetrizing variables with streamline coordinates, $\mathrm{d} \boldsymbol{\Phi}_{\mathrm{SS}}=\left(\mathrm{d} q_{1} \mathrm{~d} q_{2} \mathrm{~d} q_{3} \frac{\mathrm{d} p}{\rho c} \mathrm{~d} s\right)^{\mathrm{T}}$, defined in Appendix C.

The VLR preconditioner in the symmetrizing variables and streamline coordinates reads

$$
\mathbf{P}_{\mathrm{VLR}}^{\mathrm{ss}}=\left(\begin{array}{ccccc}
\frac{\tau}{\beta^{2}}+1 & 0 & 0 & -\frac{\tau M}{\beta^{2}} & 0 \\
0 & \tau & 0 & 0 & 0 \\
0 & 0 & \tau & 0 & 0 \\
-\frac{\tau M}{\beta^{2}} & 0 & 0 & \frac{\tau M^{2}}{\beta^{2}} & 0 \\
0 & 0 & 0 & 0 & 1
\end{array}\right)
$$


where

$$
\tau=\min \left\{\beta, \frac{\beta}{M}\right\}= \begin{cases}\beta & M<1 \\ \frac{\beta}{M} & M \geq 1\end{cases}
$$

and $\beta=\sqrt{\left|1-M^{* 2}\right|}$. In order to avoid division by zero in $(24), M^{*}$ is defined as

$$
M^{*}= \begin{cases}M & M \in\left(0,1-\epsilon_{1}\right) \cup\left(1+\epsilon_{1},+\infty\right) \\ 1-\epsilon_{1} & M \in\left(1-\epsilon_{1}, 1\right) \\ 1+\epsilon_{1} & M \in\left[1,1+\epsilon_{1}\right)\end{cases}
$$

where $\epsilon_{1}=0.01$. For the problems that we solved so far, (26) has proven to be fine. However a more sophisticated way of avoiding the sonic-point discontinuity is found in [23]. Other articles of reference for the VLR preconditioner are $[10,9,22,14,15]$. The importance of the eigenvectors structure of the preconditioned system is explained in [24]. An extension of the Euler VLR preconditioner to the Navier-Stokes equations is found in [10, 25, 26, 27].

The VLR preconditioner in the conservative variables (3) is

$$
\mathbf{P}_{\mathrm{VLR}}=\frac{\partial \Phi}{\partial \boldsymbol{\Phi}_{\mathrm{ss}}} \mathbf{P}_{\mathrm{VLR}}^{\mathrm{ss}} \frac{\partial \boldsymbol{\Phi}_{\mathrm{ss}}}{\partial \boldsymbol{\Phi}}
$$

An explicit expression of (27) is found in Appendix F, Eqs. (F.1)-(F.2).

$2 D$ condition number. In two dimensions, the characteristic propagation speeds or eigenvalues for the VLR preconditioned system are

$$
\begin{aligned}
& \lambda_{1,2}=c M n_{1} \\
& \lambda_{3,4}=\frac{1}{2} c M \tau\left[\left(1+\frac{M^{2}-1}{\beta^{2}}\right) n_{1} \pm \sqrt{\left(1+\frac{M^{2}-1}{\beta^{2}}\right)^{2} n_{1}^{2}+4 \frac{1-M^{2} n_{1}^{2}}{\beta^{2}}}\right]
\end{aligned}
$$

Using (28) into (22) (or into (E.1) from Appendix E), the group velocities of the VLR preconditioned system are

$$
\begin{aligned}
& \mathbf{g}_{1,2}=(c M, 0)^{\mathrm{T}} \\
& \mathbf{g}_{3,4}= \begin{cases} \pm \frac{c M}{\sqrt{1-M^{2} n_{1}^{2}}}\left(\left(1-M^{2}\right) n_{1}, n_{2}\right)^{\mathrm{T}} & M<1 \\
c\left(\sqrt{M^{2}-1}, \pm 1\right)^{\mathrm{T}} & M>1,\end{cases}
\end{aligned}
$$


and their norms are

$$
\begin{aligned}
& \left\|\mathbf{g}_{1,2}\right\|=c M \\
& \left\|\mathbf{g}_{3,4}\right\|= \begin{cases}c M \sqrt{\frac{1-M^{2}\left(2-M^{2}\right) n_{1}^{2}}{1-M^{2} n_{1}^{2}}} & M<1 \\
c M & M>1 .\end{cases}
\end{aligned}
$$

By Eqs. (30) and (16) the condition number is

$$
\kappa= \begin{cases}\frac{1}{\sqrt{1-M^{2}}} & M<1 \\ 1 & M>1 .\end{cases}
$$

$3 D$ condition number. Using the same definitions and following the path of the two-dimensional case, we proceed here in three dimensions. The eigenvalues of the system are

$$
\begin{aligned}
& \lambda_{1,2}=c M n_{1} \\
& \lambda_{3}=c M \tau n_{1} \\
& \lambda_{4,5}=\frac{1}{2} c M \tau\left[\left(1+\frac{M^{2}-1}{\beta^{2}}\right) n_{1} \pm \sqrt{\left(1+\frac{M^{2}-1}{\beta^{2}}\right)^{2} n_{1}^{2}+4 \frac{1-M^{2} n_{1}^{2}}{\beta^{2}}}\right]
\end{aligned}
$$

the group velocities $\mathbf{g}(\mathbf{n})$ are

$$
\begin{aligned}
& \mathbf{g}_{1,2}=(c M, 0,0)^{\mathrm{T}} \\
& \mathbf{g}_{3}= \begin{cases}\left(c M \sqrt{1-M^{2}}, 0,0\right)^{\mathrm{T}} & M<1 \\
\left(c \sqrt{M^{2}-1}, 0,0\right)^{\mathrm{T}} & M>1\end{cases} \\
& \mathbf{g}_{4,5}= \begin{cases}\left. \pm \frac{c M}{\sqrt{1-M^{2} n_{1}^{2}}}\left(1-M^{2}\right) n_{1}, n_{2}, n_{3}\right)^{\mathrm{T}} & M<1 \\
\left(\sqrt{M^{2}-1}, \pm \frac{n_{2}}{\sqrt{1-n_{1}^{2}}}, \pm \frac{n_{3}}{\sqrt{1-n_{1}^{2}}}\right)^{\mathrm{T}} & M>1,\end{cases}
\end{aligned}
$$

and their norms are

$$
\begin{aligned}
& \left\|\mathbf{g}_{1,2}\right\|=c M \\
& \left\|\mathbf{g}_{3}\right\|= \begin{cases}c M \sqrt{1-M^{2}} & M<1 \\
c \sqrt{M^{2}-1} & M>1\end{cases} \\
& \left\|\mathbf{g}_{4,5}\right\|= \begin{cases}c M \sqrt{\frac{1-M^{2}\left(2-M^{2}\right) n_{1}^{2}}{1-M^{2} n_{1}^{2}}} & M<1 \\
c M & M>1 .\end{cases}
\end{aligned}
$$


The condition number of the VLR preconditioned system is then

$$
\kappa=\frac{1}{\sqrt{1-\min \left\{M^{2}, M^{-2}\right\}}} .
$$

For both the two and three-dimensional cases, the largest eigenvalues absolute value of the VLR preconditioned system in all regimes is

$$
|\lambda|_{\max }=c M=\|u\|
$$

\subsection{CM preconditioner}

The CM preconditioner is introduced for viscous and inviscid flow in [2] using the primitive variables, $\boldsymbol{\Phi}_{\mathrm{P}}=\left(u_{1} u_{2} u_{3} p T\right)^{\mathrm{T}}$. The matrices of variable change between the primitive and the conservative variables are found in Appendix D. The CM preconditioner is not optimal (i.e.: it does not provide an optimal condition number) and its benefits only exist for low Mach numbers. However, it has the advantage to be easily adapted to viscous flow [2] and transient problems [17].

If we write the preconditioned Euler equations as

$$
\frac{\partial \boldsymbol{\Phi}_{\mathrm{P}}}{\partial t}+\boldsymbol{\Gamma}^{-1} \mathbf{A}^{i} \frac{\partial \boldsymbol{\Phi}}{\partial x_{i}}=0
$$

the $\mathrm{CM}$ preconditioner (as presented in [2]) reads

$$
\boldsymbol{\Gamma}^{-1}=\left(\begin{array}{ccccc}
\frac{1}{\rho} & 0 & 0 & \frac{-u_{1}}{\rho} & 0 \\
0 & \frac{1}{\rho} & 0 & \frac{-u_{2}}{\rho} & 0 \\
0 & 0 & \frac{1}{\rho} & \frac{-u_{3}}{\rho} & 0 \\
0 & 0 & 0 & \beta M_{r}^{2} & 0 \\
\frac{-u_{1}}{c_{p} \rho} & \frac{-u_{2}}{c_{p} \rho} & \frac{-u_{3}}{c_{p} \rho} & \frac{1}{c_{p} \rho}\left(\frac{1}{2}\|\mathbf{u}\|^{2}-c_{p} T+\beta M_{r}^{2}\right) & \frac{1}{c_{p} \rho}
\end{array}\right) ;
$$

its inverse is

$$
\boldsymbol{\Gamma}=\left(\begin{array}{ccccc}
\rho & 0 & 0 & \frac{u_{1}}{\beta M_{r}^{2}} & 0 \\
0 & \rho & 0 & \frac{u_{2}}{\beta M_{r}^{2}} & 0 \\
0 & 0 & \rho & \frac{u_{3}}{\beta M_{r}^{2}} & 0 \\
0 & 0 & 0 & \frac{1}{\beta M_{r}^{2}} & 0 \\
\rho u_{1} & \rho u_{2} & \rho u_{3} & \frac{1}{\beta M_{r}^{2}} \frac{E+p}{\rho} & c_{p} \rho
\end{array}\right),
$$


where $\beta=k_{\beta} c^{2}, k_{\beta}=1$ for inviscid problems, and

$$
M_{r}= \begin{cases}\epsilon_{0} & M<\epsilon_{0} \\ M & \epsilon_{0}<M<1 \\ 1 & 1<M\end{cases}
$$

where $\epsilon_{0}=10^{-5}$, as it is defined in [2]. For the problems that we solved so far, (40) has proven to be fine.

The CM preconditioner in the conservative variables (3) is

$$
\mathbf{P}_{\mathrm{CM}}=\frac{\partial \boldsymbol{\Phi}}{\partial \boldsymbol{\Phi}_{\mathrm{P}}} \boldsymbol{\Gamma}^{-1}
$$

An explicit expression of (41) is given by Eq. (G.1), in Appendix G. The CM preconditioner in the symmetrizing variables and streamline coordinates is

$$
\mathbf{P}_{\mathrm{CM}}^{\mathrm{ss}}=\frac{\partial \boldsymbol{\Phi}_{\mathrm{ss}}}{\partial \boldsymbol{\Phi}} \mathbf{P}_{\mathrm{CM}} \frac{\partial \boldsymbol{\Phi}}{\partial \boldsymbol{\Phi}_{\mathrm{ss}}} .
$$

An explicit expression of (42) is found in Eq. (H.1) from Appendix H.

$2 D$ and $3 D$ condition numbers. The eigenvalues of the CM preconditioned system are

$$
\begin{aligned}
& \lambda_{1, \ldots, d}=c M n_{1} \\
& \lambda_{d+1, d+2}=\frac{1}{2} c\left(M n_{1}\left(1+M_{r}^{2}\right) \pm \sqrt{M^{2} n_{1}^{2}\left(1+M_{r}^{2}\right)^{2}+4 M_{r}^{2}\left(1-M^{2} n_{1}^{2}\right)}\right)
\end{aligned}
$$

where $\mathbf{n}$ is a unit vector defining the wave propagation direction. As it is pointed out in [22], above Mach numbers of approximately 0.4, the CM preconditioner has worse condition number than the unpreconditioned Euler equations. When $M>1$ we have $M_{r}=1$ and we found the same eigenvalues as for the non-preconditioned case. For this reason CM is a preconditioner for low Mach number regimes and it could be switched off when $M>0.4$. The maximum eigenvalue in absolute value in all regimes is

$$
|\lambda|_{\max }=\frac{1}{2} c\left(M\left(1+M_{r}^{2}\right)+\sqrt{M^{2}\left(1+M_{r}^{2}\right)^{2}+4 M_{r}^{2}\left(1-M^{2}\right)}\right) .
$$

Taking definition (22) (or equivalently, expressions (E.1)-(E.4) from Appendix E) and using 
the values of (43), the group velocities are

$$
\begin{aligned}
& \mathbf{g}_{1, \ldots, d}=(c M, 0, \ldots, 0)^{\mathrm{T}} \\
& \mathbf{g}_{d+1, d+2}= \begin{cases}\frac{1}{2} c\left(M\left(1+M_{r}^{2}\right) \pm n_{1} \frac{M^{2}\left(1-M_{r}^{2}\right)^{2}+4 M_{r}^{2}}{\sqrt{M^{2} n_{1}^{2}\left(1-M_{r}^{2}\right)^{2}+4 M_{r}^{2}}}, \pm n_{2} \frac{4 M_{r}^{2}}{\sqrt{M^{2} n_{1}^{2}\left(1-M_{r}^{2}\right)^{2}+4 M_{r}^{2}}}, \ldots,\right. & \\
\left. \pm n_{d} \frac{4 M_{r}^{2}}{\sqrt{M^{2} n_{1}^{2}\left(1-M_{r}^{2}\right)^{2}+4 M_{r}^{2}}}\right)^{\mathrm{T}} & M<1 \\
\left(M \pm n_{1}, n_{2}, \ldots, n_{d}\right)^{\mathrm{T}} & M>1\end{cases}
\end{aligned}
$$

and their norms are

$$
\begin{aligned}
& \left\|\mathbf{g}_{1, \ldots, d}\right\|=c M \\
& \left\|\mathbf{g}_{d+1, d+2}\right\|= \begin{cases}\frac{1}{2} c\left[\left(M\left(1+M_{r}^{2}\right) \pm \frac{\left(M^{2}\left(1-M_{r}^{2}\right)^{2}+4 M_{r}^{2}\right) n_{1}}{\sqrt{M^{2} n_{1}^{2}\left(1-M_{r}^{2}\right)^{2}+4 M_{r}^{2}}}\right)^{2}+\right. & \\
\left.\left(\frac{4 M_{r}^{2}\left(1-n_{1}^{2}\right)}{\sqrt{M^{2} n_{1}^{2}\left(1-M_{r}^{2}\right)^{2}+4 M_{r}^{2}}}\right)^{2}\right]^{\frac{1}{2}} & M<1 \\
c \sqrt{M^{2}+1 \pm 2 M n_{1}} & M>1 .\end{cases}
\end{aligned}
$$

Then, from (46) and definition (16), the condition number of the CM preconditioned system is

$$
\kappa= \begin{cases}\frac{M\left(1+M_{r}^{2}\right)+\sqrt{M^{2}\left(1-M_{r}^{2}\right)^{2}+4 M_{r}^{2}}}{\mid M\left(1+M_{r}^{2}\right)-\sqrt{M^{2}\left(1-M_{r}^{2}\right)^{2}+4 M_{r}^{2}}} & M<1 \\ \frac{M+1}{M-1} & M>1 .\end{cases}
$$

\section{Numerical formulation}

In this section we set the bases for the P-VMS method to solve the compressible Euler equations. The preconditioned equations (20) are discretized in space using finite elements with VMS stabilization and, in time, using a first order forward finite difference scheme.

\subsection{Finite element space discretization}

Let $\Omega^{h}$ be a polyhedral approximation of $\Omega$, the domain of the continuous problem (20). We consider a partition $\mathcal{P}^{h}=\left\{K^{m}\right\}_{m=1, \ldots, N_{e l}}$ of $\Omega^{h}$ in $N_{e l}$ elements, $K^{m} \subset \Omega^{h}$, of characteristic length 


\begin{tabular}{|c|c|c|c|}
\hline \multirow{3}{*}{ 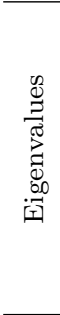 } & 号 & & $\left\{c M n_{1}, c M n_{1}, c\left(M n_{1} \pm 1\right)\right\}$ \\
\hline & $\stackrel{2}{\frac{2}{3}}$ & $\begin{array}{l}M<1 \\
M>1\end{array}$ & $\begin{array}{l}\left.\left\{c M n_{1}, c M n_{1}, \pm c M \sqrt{1-M^{2} n_{1}^{2}}\right)\right\} \\
\left\{c M n_{1}, c M n_{1}, c\left(\sqrt{M^{2}-1} n_{1} \pm \sqrt{1-n_{1}^{2}}\right)\right\}\end{array}$ \\
\hline & $\sum_{U}$ & $\begin{array}{l}M<1 \\
M>1\end{array}$ & $\begin{array}{l}\left\{c M n_{1}, c M n_{1}, \frac{1}{2} c\left(M n_{1}\left(1+M_{r}^{2}\right) \pm \sqrt{M^{2} n_{1}^{2}\left(1+M_{r}^{2}\right)^{2}+4 M_{r}^{2}\left(1-M^{2} n_{1}^{2}\right)}\right)\right\} \\
\left\{c M n_{1}, c M n_{1}, c\left(M n_{1} \pm 1\right)\right\}\end{array}$ \\
\hline \multirow{3}{*}{ 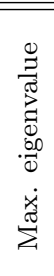 } & 号 & & $c(M+1)$ \\
\hline & $\stackrel{5}{5}$ & & $c M$ \\
\hline & $\sum_{U}$ & $\begin{array}{l}M<1 \\
M>1\end{array}$ & $\begin{array}{l}\frac{1}{2} c\left(M\left(1+M_{r}^{2}\right)+\sqrt{M^{2}\left(1+M_{r}^{2}\right)^{2}+4 M_{r}^{2}\left(1-M^{2}\right)}\right) \\
c(M+1)\end{array}$ \\
\hline \multirow{3}{*}{ 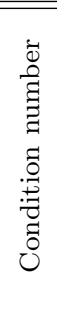 } & 号 & & $\frac{M+1}{\min \{M,|M-1|\}}$ \\
\hline & $\stackrel{2}{3}$ & $\begin{array}{l}M<1 \\
M>1\end{array}$ & $\begin{array}{l}\frac{1}{\sqrt{1-M^{2}}} \\
1\end{array}$ \\
\hline & $\sum_{0}$ & $\begin{array}{l}M<1 \\
M>1\end{array}$ & $\begin{array}{l}\frac{\sqrt{M^{2}\left(1+M_{r}^{2}\right)^{2}+4 M_{r}^{2}\left(1-M^{2}\right)}+M\left(1+M_{r}^{2}\right)}{\sqrt{M^{2}\left(1+M_{r}^{2}\right)^{2}+4 M_{r}^{2}\left(1-M^{2}\right)}-M\left(1+M_{r}^{2}\right)} \\
\frac{M+1}{M-1}\end{array}$ \\
\hline
\end{tabular}

Table 2: 2D eigenvalues, their maximum, and the condition number of the NP (non preconditioned), VLR, and CM preconditioning options.

$h^{m}$. In this work, $h^{m}$ is defined as the shortest edge of the element. Let $\left\{\mathbf{x}^{p}\right\}_{p=1, \ldots, N}$ be the $N$ nodes of the grid associated to $\mathcal{P}^{h}$ and $\psi_{p}^{h}$ the Lagrange polynomial corresponding to node $\mathbf{x}^{p}$, for $p=1, \ldots, N$. We consider $W^{h}$, the functions space generated by $\left\{\psi_{p}^{h}\right\}_{p=1, \ldots, N}$, and project the system (20) onto $W^{h}$ by the $L^{2}$ scalar product, where $L^{2}$ is the space of square-integrable real-valued functions. In this way we obtain the finite elements weak form of (20) as

$$
\int_{\Omega^{h}} \psi^{h} \frac{\partial \boldsymbol{\Phi}^{h}}{\partial t} d \Omega^{h}+\int_{\Omega^{h}} \psi^{h} \mathbf{P A}^{i}(\mathbf{\Phi}) \frac{\partial \boldsymbol{\Phi}^{h}}{\partial x_{i}} d \Omega^{h}=0 \quad \forall \psi^{h} \in W^{h} .
$$

The function $\boldsymbol{\Phi}^{h}$ is defined as the projection of $\boldsymbol{\Phi}$ onto $W^{h}$ and it can be expressed as

$$
\boldsymbol{\Phi}^{h}(\xi, t)=\sum_{p=1}^{N} \psi_{p}^{h}(\xi) \boldsymbol{\Phi}_{p}^{h}(t),
$$

where $\xi \in \Omega^{h}$ and $\boldsymbol{\Phi}_{p}^{h}(t)$ is the value of $\boldsymbol{\Phi}^{h}$ at nodes $\mathbf{x}^{p}$ and time $t$. 


\begin{tabular}{|c|c|c|c|}
\hline \multirow{3}{*}{ 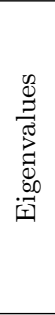 } & 号 & & $\left\{c M n_{1}, c M n_{1}, c M n_{1}, c\left(M n_{1} \pm 1\right)\right\}$ \\
\hline & $\stackrel{r}{s}$ & $\begin{array}{l}M<1 \\
M>1\end{array}$ & $\begin{array}{l}\left.\left\{c M n_{1}, c M n_{1}, c M \sqrt{1-M^{2}} n_{1}, \pm c M \sqrt{1-M^{2} n_{1}^{2}}\right)\right\} \\
\left\{c M n_{1}, c M n_{1}, c \sqrt{M^{2}-1} n_{1}, c\left(\sqrt{M^{2}-1} n_{1} \pm \sqrt{1-n_{1}^{2}}\right)\right\}\end{array}$ \\
\hline & $\sum$ & $\begin{array}{l}M<1 \\
M>1\end{array}$ & $\begin{array}{l}\left\{c M n_{1}, c M n_{1}, c M n_{1}, \frac{1}{2} c\left(M n_{1}\left(1+M_{r}^{2}\right) \pm \sqrt{M^{2} n_{1}^{2}\left(1+M_{r}^{2}\right)^{2}+4 M_{r}^{2}\left(1-M^{2} n_{1}^{2}\right)}\right)\right\} \\
\left\{c M n_{1}, c M n_{1}, c M n_{1}, c\left(M n_{1} \pm 1\right)\right\}\end{array}$ \\
\hline \multirow{3}{*}{ 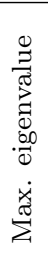 } & 号 & & $c(M+1)$ \\
\hline & $\frac{5}{5}$ & & $c M$ \\
\hline & $\sum_{0}$ & $\begin{array}{l}M<1 \\
M>1\end{array}$ & $\begin{array}{l}\frac{1}{2} c\left(M\left(1+M_{r}^{2}\right)+\sqrt{M^{2}\left(1+M_{r}^{2}\right)^{2}+4 M_{r}^{2}\left(1-M^{2}\right)}\right) \\
c(M+1)\end{array}$ \\
\hline \multirow{3}{*}{ 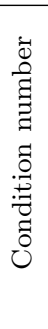 } & 吕 & & $\frac{M+1}{\min \{M,|M-1|\}}$ \\
\hline & 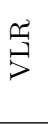 & $\begin{array}{l}M<1 \\
M>1\end{array}$ & $\begin{array}{l}\frac{1}{\sqrt{1-M^{2}}} \\
\frac{M}{\sqrt{M^{2}-1}}\end{array}$ \\
\hline & $\sum_{U}$ & $\begin{array}{l}M<1 \\
M>1\end{array}$ & $\begin{array}{l}\frac{\sqrt{M^{2}\left(1+M_{r}^{2}\right)^{2}+4 M_{r}^{2}\left(1-M^{2}\right)}+M\left(1+M_{r}^{2}\right)}{\sqrt{M^{2}\left(1+M_{r}^{2}\right)^{2}+4 M_{r}^{2}\left(1-M^{2}\right)}-M\left(1+M_{r}^{2}\right)} \\
\frac{M+1}{M-1}\end{array}$ \\
\hline
\end{tabular}

Table 3: 3D eigenvalues, its maximum and the condition number of the NP (non preconditioned), VLR, and CM preconditioning options.

\subsection{P-VMS: Stabilization by the variational multiscale method}

When convection dominates, as it is the case of the Euler equations, the finite elements discretization (48) presents some instabilities that should be treated in order to avoid non-physical oscillations or blow-up of the solution [28]. These instabilities can be treated by means of different stabilization procedures. Pioneering work on stabilization techniques for finite elements for compressible flows are the Streamline Upwind Petrov-Galerkin (SUPG) [29, 30, 31, 32] and the Galerkin/Least-Squares (GLS) [33] methods. A complete survey of both stabilization procedures can be found in [34].

The unresolved scales is the part of the solution that is under the resolution of the grid and consequently can not be solved by the numerical method. In the 90's a group of researchers lead by Hughes state that the instabilities come from the fact that its effect on the solution is not captured by the numerical method. They conclude that the effects of the unresolved scales must be introduced in the formulation of the discrete problem, by modeling them in some way, using 
the information that we have at the grid scale level. Based on this idea they designed a new stabilization framework, the so-called variational multiscale stabilization method. VMS was firstly introduced for the advection-diffusion equations in $[35,36]$. This approach allows the development of new schemes but also the understanding of previous stabilization techniques as SUPG and GLS.

VMS has been widely applied to the incompressible equations, being its application to compressible flow problems more recent. To our knowledge, VMS for compressible flows appears in [37, 38, 39, 40, 41, 19]. Hybrid VMS-LES approaches for large eddy simulation of turbulent compressible flows can be found in $[42,43,44,45,46,47]$. Due to its high efficiency, robustness, and validity at all Mach regimes, in this paper we focus on the variational multiscale approach. The method introduced in [19] is used in this paper and adapted for the preconditioned problem (20).

A fundamental concept of VMS is that the unknown consists of two components, the large scale $\mathbf{\Phi}^{h}$, and the unresolved part or subscale $\tilde{\mathbf{\Phi}}$ :

$$
\boldsymbol{\Phi}=\boldsymbol{\Phi}^{h}+\tilde{\boldsymbol{\Phi}} .
$$

The large scale is the part of the unknown that is solved explicitly, while the subscales and their effect on the large scale problem (48), are modeled by the VMS method. Introducing split (50) in Eq. (48), we obtain the VMS stabilized problem: $\forall \psi^{h} \in W^{h}$,

$$
\int_{\Omega^{h}} \psi^{h} \frac{\partial \boldsymbol{\Phi}^{h}}{\partial t} d \Omega^{h}+\int_{\Omega^{h}} \psi^{h} \mathbf{P A}^{i}(\mathbf{\Phi}) \frac{\partial \boldsymbol{\Phi}^{h}}{\partial x_{i}} d \Omega^{h}+\sum_{K \in \mathcal{P}_{h}} \int_{K} \psi^{h}\left(\frac{\partial \tilde{\boldsymbol{\Phi}}}{\partial t}+\mathbf{P A}^{i}(\mathbf{\Phi}) \frac{\partial \tilde{\mathbf{\Phi}}}{\partial x_{i}}\right) d K=0 .
$$

The effect of the subscale on the large scale is represented by the stabilization term, consisting of the last two terms in Eq. (51). We use $\sum_{K \in \mathcal{P}_{h}} \int_{K}$ instead of $\int_{\Omega^{h}}$ in the stabilization term of (51) because the subscales are defined inside the elements but not necessarily on their boundaries. In what follows, we enumerate some approximations that we consider in Eq. (51). (A) We consider orthogonal subscales, as it is done in $[48,49]$ in the context of advection-diffusion and incompressible flow equations. Under this assumption, $\int_{K} \psi^{h} \frac{\partial \tilde{\Phi}}{\partial t} d K=0$. (B) In the interest of algorithmic simplicity, we make the approximation $\mathbf{P A}(\boldsymbol{\Phi}) \approx \mathbf{P} A^{i}\left(\boldsymbol{\Phi}^{h}\right)$ and simply write $\mathbf{P} A^{i}$ from now on. This approximation is usually done among VMS practitioners (see, for example, [37]) and implicitly done in the case of SUPG or GLS stabilization, where the concept of subscale and the decomposition (50) do not exist. In the context of incompressible flow, the exact form $\mathbf{P} \mathbf{A}^{i}(\boldsymbol{\Phi})$ is preserved in [49]; this requires the storage of the subscale at each iteration. (C) As it is commonly done in the VMS community, in order to avoid the space derivatives of the subscale, we integrate by parts 
the last term of (51) and we suppose the arising boundary term to be zero. Finally, consider that the test functions space $W^{h}$ is generated by $\left\{\psi_{p}^{h}\right\}_{p=1, \ldots, N}$. Then, imposing Eq. (51) to hold for all $\psi^{h} \in W^{h}$ is equivalent to imposing it to hold for all $\psi_{p}^{h}, p \in\{1, \ldots, N\}$. The last assumptions and considerations lead to the reformulation of (51) as:

$$
\underbrace{\int_{\Omega^{h}} \psi_{p}^{h} \frac{\partial \boldsymbol{\Phi}^{h}}{\partial t} d \Omega^{h}+\int_{\Omega^{h}} \psi_{p}^{h} \mathbf{P A}^{i} \frac{\partial \mathbf{\Phi}^{h}}{\partial x_{i}} d \Omega^{h}}_{\text {Galerkin term }}-\underbrace{-\sum_{K \in \mathcal{P}_{h}} \int_{K}\left(\frac{\partial \psi_{p}^{h}}{\partial x_{i}} \mathbf{P A}^{i}+\psi_{p}^{h} \frac{\partial\left(\mathbf{P A}^{i}\right)}{\partial x_{i}}\right) \tilde{\mathbf{\Phi}} d K}_{\text {Stabilization term }}=0,
$$

holding for all $p \in\{1, \ldots, N\}$. A model for the subscales is explicitly defined in the paragraph Subscale approximation below. Eq. (52) is the weak stabilized form of Eq. (20). In the current implementation of the method, we neglect term $\int_{K} \psi_{p}^{h} \frac{\partial\left(\mathbf{P A}^{i}\right)}{\partial x_{i}} \tilde{\mathbf{\Phi}} d K$ in Eq. (52). This simplification is standard, however we should consider this term in future implementations of P-VMS. We considered this term in [19] in the context of VMS, when solving the Euler and Navier-Stokes equations without preconditioning. As far as we know, [19] is the only reference of VMS where this term is taken into account. The neglected term neither appears in the SUPG or GLS formulations.

Subscale approximation. The subscales are approximated in every element $K^{m} \in \mathcal{P}^{h}$ as the product of a parameter matrix $\tau$ times the residual of the governing equation:

$$
\tilde{\boldsymbol{\Phi}}=\tau \mathbf{r}\left(\boldsymbol{\Phi}^{h}\right)
$$

where

$$
\mathbf{r}\left(\boldsymbol{\Phi}^{h}\right)=-\mathbf{P A}^{j} \frac{\partial \boldsymbol{\Phi}^{h}}{\partial x_{j}} .
$$

It is important to see the local nature of the subscales that are meant to exist only where residuals are important. Expression (53) is plugged into Eq. (52) to find an expression of the variational multiscale stabilized finite element method for Eq. (20). Most of the models describe the subscale in the form of Eq. (53). However their residual includes the time derivative term. This term does not appear in our residual (54) because we are considering orthogonal subscales (see assumption (A) above). The definition of the parameter $\tau$ is a topic of active research still today. There exist, for compressible flow, many proposals in the literature. Some definitions for the stabilization parameter $\tau$ are found in $[29,30,31]$, in the context of SUPG; in [33, 50], in the context of GLS; and 
in [37], in the context of VMS. The stabilization parameter $\tau$ is here defined inside each element as

$$
\tau=\frac{h}{|\lambda|_{\max }}
$$

where $h$ is chosen as the smallest edge length of the element and $|\lambda|_{\max }$ is defined in (21) (in (10) for the non-preconditioned case). Using expression (36) for the VLR case, (55) becomes

$$
\tau=\frac{h}{\|\mathbf{u}\|} .
$$

The same parameter $\tau$ (56) will be used for the CM preconditioned case. Let's call $\tau_{\mathrm{CM}}$ the parameter (55) defined using $|\lambda|_{\max }$ from expression (44). After numerical experiments, parameter (56) is shown to perform better for the CM preconditioned case than $\tau_{\mathrm{CM}}$. We observe that $\tau_{\mathrm{CM}}$ is always smaller than (56). This could be a reason for that phenomena that, however, is not completely clear. In (56), if $\mathbf{u}=0$ a lower bound is used. Using expression (13) for the unpreconditioned case, we get

$$
\tau=\frac{h}{\|\mathbf{u}\|+c}
$$

where $c$ is the speed of sound. In the unpreconditioned case, we find in the literature alternative definitions of the parameter $\tau$ which are also based on the eigenvalues of the Jacobians $\mathbf{A}^{i}$ and depend on the speed of sound. See for example $[29,30,31,33,50]$.

\subsection{Time discretization. Global and local time stepping}

A first order explicit time discretization of (52) gives

$$
\int_{\Omega^{h}} \psi_{p}^{h} \frac{\boldsymbol{\Phi}^{h, n+1}-\boldsymbol{\Phi}^{h, n}}{\Delta t} d \Omega^{h}+\int_{\Omega^{h}} \psi_{p}^{h} \mathbf{P} \mathbf{A}^{i} \frac{\partial \boldsymbol{\Phi}^{h, n}}{\partial x_{i}} d \Omega^{h}-\sum_{K \in \mathcal{P}_{h}} \int_{K} \frac{\partial \psi_{p}^{h}}{\partial x_{i}} \mathbf{P A}^{i} \tilde{\mathbf{\Phi}}^{n+1} d K=0,
$$

holding for all $p \in\{1, \ldots, N\}$. The superscripts $n+1$ and $n$ indicate the value at the current and the previous time step, respectively. The value of $\tilde{\boldsymbol{\Phi}}^{n+1}$ is computed from (53) using the information of the previous time step, i.e.: $\tilde{\boldsymbol{\Phi}}^{n+1}=\tau^{n} \mathbf{r}\left(\boldsymbol{\Phi}^{h, n}\right)$.

In this paper, both local and global time stepping are considered. From the CFL condition [21] a time step inside each element is defined as

$$
\Delta t^{e l}=C \frac{h}{|\lambda|_{\max }},
$$


where $C \in(0,1)$ is the CFL number, $h$ is the smallest edge length of the element, and $|\lambda|_{\text {max }}$ is the maximum eigenvalue in absolute value, defined by (21) and (10) for the preconditioned and the non-preconditioned cases, respectively.

The elementary time step (59) is interpolated on the nodes of the grid, obtaining a local time step $\Delta t^{p}, p=1, \ldots, N$, at each node $\mathbf{x}^{p}$. When using a local time stepping, the solution at each node of the grid advances in time with its own time step, $\Delta t^{p}$, computed with local parameters. Then, the local time step is different at each node of the grid and it is the same for all the equations of our system. We note that no time synchronization is needed since the steady solution is sought.

When using a global time stepping, we advance in time the solution with a unique time step for the whole domain. The global time step is computed as the minimum time step of the domain as

$$
\Delta t=\min _{p=1, \ldots, N}\left\{\Delta t^{p}\right\} .
$$

For the VLR preconditioner, we use (36) in Eq. (59) and the elementary time step becomes

$$
\Delta t^{e l}=C \frac{h}{\|\mathbf{u}\|} .
$$

The same time step (61) is here used for the CM preconditioned case, as it is seen to perform better than the one using its own $|\lambda|_{\max }$, defined in (44). The same phenomena happened with the $\tau$ parameter definition (56) for the CM case. In (61), if $\mathbf{u}=0$ a lower bound is used to avoid division by zero. Using expression (13), the time step for the unpreconditioned case is computed inside each element as

$$
\Delta t^{e l}=C \frac{h}{\|\mathbf{u}\|+c} .
$$

From the definitions, it can be observed that, especially in low-Mach regimes, the time step for the preconditioned case could be orders of magnitude larger than the non-preconditioned one. It will be shown in the numerical results that this fact entails a speed up of the convergence for the preconditioned system.

Inserting equality (49) in (58), a linear system of $N(d+2)$ equations is obtained:

$$
\mathbf{M} \frac{\boldsymbol{\Phi}^{h, n+1}-\boldsymbol{\Phi}^{h, n}}{\Delta t}=\mathbf{G}\left(\boldsymbol{\Phi}^{h, n}\right)+\mathbf{S}\left(\boldsymbol{\Phi}^{h, n}, \tilde{\boldsymbol{\Phi}}^{n+1}\right),
$$

where 
1. $\boldsymbol{\Phi}^{h}$ is here the nodal vector of unknowns of dimension $N(d+2)$. It is made by assembly of the vectors $\boldsymbol{\Phi}_{p}^{h}$, for $p=1, \ldots, N$, where $\boldsymbol{\Phi}_{p}^{h}$ is $\boldsymbol{\Phi}^{h}$ evaluated at node $\mathbf{x}^{p}$.

2. $\mathbf{M}$ is the global Mass matrix, it has dimension $N(d+2) \times N(d+2)$. It is a block matrix composed of $N^{2}$ blocks $\mathbf{M}^{p q}$ of dimension $(d+2) \times(d+2)$,

$$
\mathbf{M}^{p q}=\int_{\Omega^{h}} \psi_{p}^{h} \psi_{q}^{h} d \Omega^{h} \mathbf{I}_{d+2},
$$

where $p, q=1, \ldots, N$ and $\mathbf{I}_{d+2}$ is the identity matrix of dimension $(d+2) \times(d+2)$.

3. $\mathbf{G}$ and $\mathbf{S}$ are the Galerkin and stabilization vector terms, respectively. They are constructed by assembly of $N$ local vectors of dimension $d+2$ :

$$
\mathbf{G}^{p}=-\int_{\Omega^{h}} \psi_{p}^{h} \mathbf{P A}^{i} \frac{\partial \boldsymbol{\Phi}^{h, n}}{\partial x_{i}} d \Omega^{h} \quad \mathbf{S}^{p}=\sum_{K \in \mathcal{P}_{h}} \int_{K} \frac{\partial \psi_{p}^{h}}{\partial x_{i}} \mathbf{P A}^{i} \tilde{\boldsymbol{\Phi}}^{n+1} d K,
$$

respectively, for $p=1, \ldots, N$.

All the integrals above are approximated by the Gaussian quadrature rule that, for a function $f$, writes

$$
\int_{K} f(\mathbf{x}) d K=\int_{I} f(\mathcal{H}(\xi))|J(\xi)| d \xi \approx \sum_{p=1}^{N_{\text {Gauss }}} f\left(\mathcal{H}\left(\xi^{p}\right)\right)\left|J\left(\xi^{p}\right)\right| \omega^{p},
$$

where $I=[-1,1] \times[-1,1]$ is a reference element, $\mathcal{H}$ a bijection from $I$ to the element $K, J=\frac{\mathrm{d} \mathcal{H}}{\mathrm{d} \xi}$ the $\mathcal{H}$ Jacobian matrix, $|J|$ its determinant, $N_{\text {Gauss }}$ is the number of integration Gauss points $\xi^{p}$ inside the element $K$, and $\omega^{p}$ its weight.

$\mathbf{M}$ is diagonalized by lumping techniques [51] in order to avoid its inversion when open integration rules are used. From Eq. (63), the value of $\boldsymbol{\Phi}^{h, n+1}$ at each node of the computational grid is obtained as

$$
\boldsymbol{\Phi}^{h, n+1}=\boldsymbol{\Phi}^{h, n}+\Delta t \mathbf{M}^{-1}\left(\mathbf{G}\left(\boldsymbol{\Phi}^{h, n}\right)+\mathbf{S}\left(\boldsymbol{\Phi}^{h, n}, \tilde{\mathbf{\Phi}}^{n+1}\right)\right)
$$

where we defined $\tilde{\boldsymbol{\Phi}}^{n+1}=\tau \mathbf{r}\left(\boldsymbol{\Phi}^{h, n}\right)$ and $\boldsymbol{\Phi}^{h, n}$ is computed using equality (49).

\section{Numerical results}

P-VMS performance is assessed through two well-known benchmarks for inviscid flow problems. The first example is the flow past a two dimensional NACA 0012 airfoil at zero angle of attack 
and different Mach numbers, from subsonic flows at very low Mach numbers, up to supersonic regimes. The second one is the three dimensional ONERA M6 wing test case at $3.06^{\circ}$ angle of attack and a Mach number of 0.8395. A first order explicit time integration scheme is used for the time integration. The presented scheme is implemented in BSC's in-house code: Alya.

VLR and CM preconditioners are used and compared to the non preconditioned (NP) case. For these three preconditioning options (NP, VMS, and CM), both global and local time stepping are tested, giving the following options:

- No preconditioning with global time step (NP-globalTS)

- No preconditioning with local time step (NP-localTS)

- VLR preconditioning with global time step (VLR-globalTS)

- VLR preconditioning with local time step (VLR-localTS)

- CM preconditioning with global time step (CM-globalTS)

- $\mathrm{CM}$ preconditioning with local time step (CM-localTS)

In what follows, when we want to specify the time stepping that is used, we will refer to the different cases by using the above abbreviations in brackets. At the continuous stage the unpreconditioned and the preconditioned equations, (4) and (20), respectively, share the same steady solution. However, both systems are different and have different condition numbers, so that they can react differently to the numerical discretization. Consequently they can give slightly different solutions, or one of them can converge while the other diverges. As noted in [22], local preconditioning "[...] has the benefit of increasing the accuracy of the discretization". This fact would be seen through the following test cases.

For both test cases, a constant velocity and temperature are prescribed at the inflow and a constant density at the outflow. The distance from the airfoil to the inflow and outflow boundaries is taken large enough in order to avoid the numerical instabilities of reflecting waves. Slip boundary conditions are used on the airfoil and on the walls.

Convergence rates are here represented as a normalized residual on the vertical axis over the 
iteration number on the horizontal axis. We call normalized residual the quantity Res defined as:

$$
\begin{aligned}
& R e s=\frac{\|\Delta \mathbf{U}\|}{\|\mathbf{U}\|}+\frac{\|\Delta \rho\|}{\|\rho\|}+\frac{\|\Delta E\|}{\|E\|},
\end{aligned}
$$

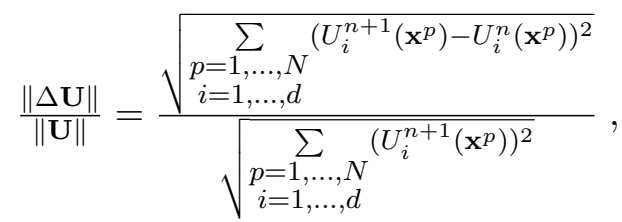

$$
\begin{aligned}
& \frac{\|\Delta \rho\|}{\|\rho\|}=\frac{\sqrt{\sum_{p=1, \ldots, N}\left(\rho^{n+1}\left(\mathbf{x}^{p}\right)-\rho^{n}\left(\mathbf{x}^{p}\right)\right)^{2}}}{\sqrt{\sum_{p=1, \ldots, N}\left(\rho^{n+1}\left(\mathbf{x}^{p}\right)\right)^{2}}}, \\
& \frac{\|\Delta E\|}{\|E\|}=\frac{\sqrt{\sum_{p=1, \ldots, N}\left(E^{n+1}\left(\mathbf{x}^{p}\right)-E^{n}\left(\mathbf{x}^{p}\right)\right)^{2}}}{\sqrt{\sum_{p=1, \ldots, N}\left(E^{n+1}\left(\mathbf{x}^{p}\right)\right)^{2}}} .
\end{aligned}
$$

\subsection{NACA 0012 airfoil}

Inviscid flow past a two-dimensional NACA 0012 airfoil is solved at zero angle of attack and inflow Mach numbers: $0.001,0.01,0.1,0.3,0.5,0.7,0.9,1.0,1.2,1.8,2.0$. The rest of the initial conditions being: $\rho=1, \mathbf{u}=(1,0), T=1$, and $c_{\mathrm{p}}$ and $c_{\mathrm{v}}$ are determined to obtain the desired inflow Mach numbers. An unstructured grid of 4522 triangles and 2315 grid nodes is used. The CFL number values used for these simulations are in Table 5. It corresponds to the maximum CFL number that can be used for each simulation, obtained through numerical experiments.

The same problem for a Mach number of 0.0001 is solved in the original paper by Choi and Merkle [2] using central differencing in space. In [1], they solve it by finite volumes, using the VLR preconditioner and different Mach numbers ranging between 0.01 and 1.8.

Results of pressure contours for the different inflow Mach numbers are displayed in Figs. 1 to 11. They show the final steady state solution corresponding to the NP and VLR options using local time stepping. The results using the CM preconditioner are not shown here, they are very similar to the VLR ones for low Mach numbers $(M \leq 0.3)$ and to the NP ones otherwise. CM is in fact a preconditioner for low Mach number regimes, otherwise it does not remove the stiffness of the non-preconditioned Euler equations. The CM preconditioned system is worse conditioned than the non-preconditioned case when $0.4<M<1$. When $M>1$ the characteristic speeds of the CM preconditioned system are indeed the same of the NP case (see Subsection 4.2 for more detail). For all Mach numbers, VLR using global and local time stepping give identical steady 
results. NP-globalTS and NP-localTS options give very similar but not identical results, the same happens between CM-globalTS and CM-localTS options.

For Mach numbers $0.001,0.01$, and 0.1 we obtain wrong results without preconditioning. However using both VLR or CM, the problem converges rapidly to a smooth solution. This is seen from Figs. 1-3. This fact shows that the use of VLR or CM preconditioners gives enough stability to VMS to handle low Mach number flows accurately and robustly.

As expected, non-preconditioned VMS becomes more stable as the Mach number increases. When $M=0.3$ the non-preconditioned VMS pressure contours still present some error at the surface of the airfoil (Fig. 4), which is not at all the case for the P-VMS in either VLR or CM. With a more refined grid the non-preconditioned VMS could give a good result, however the object of this study is to compare the convergence and stability properties of non-preconditioned and preconditioned options, under the same conditions. For Mach numbers 0.5 and 0.7 (Figs. 5 and 6) all the options give very similar results.

To better compare, no shock-capturing diffusion is used for the supersonic cases. Then the result presents some oscillations near the shocks in the supersonic regions. When $M=0.9$ (Fig. 7) VLR option seems to capture the sharp shock that appears in the tail of the airfoil more accurately than NP and CM options. We Observe in Fig. 9 that for $M=1.2$ the results of NP and CM options are different from VLR one. The front shock is formed at $x=0.5$ in the VLR case and slightly before in the NP and CM ones. The VLR pressure contours and position of the front shock compare better with the result from [52] than the NP and CM ones. Similar discrepancy between NP/CM and VLR options concerning the front shock position appears for Mach numbers 1.8 and 2.0 (Figs. 10 and 11).

The maximum and minimum values of the Mach number of the steady solution for the different preconditioning and time stepping options are displayed in Table 4 for comparison. The minimum value of the Mach number is reached at the leading edge where $M$ should be zero. This value represents the main disagreement between NP, VLR and CM options. The NP and CM cases always have larger minimum values than the VLR option. In the CM case those values are at most one order of magnitude larger than the VLR case. However, in the NP case they are most of the times one or two orders of magnitude larger than in the VLR case. This shows the improved accuracy properties of the P-VMS, especially in the case of the VLR preconditioner, for which the 
Mach number at the leading edge approaches zero the most. When $M=1.2$, the Mach number result for the NP-localTS option presents an overshot on a point of the outflow boundary, which is reflected in Table 4 in the Mach number maximum value for this case. The use of local time stepping is not as robust for the unpreconditioned case as it is when preconditioning is used.

We observe from Table 5 that the CFL number has to be reduced when a local time stepping is used. However local time stepping improves significantly the convergence rate compared to global time stepping, for all the preconditioning options. This improvement is less significant when $M \rightarrow 0$ and for the CM option when $M \rightarrow 1$. The convergences for the global time stepping are not shown here for the sake of brevity.

Results of convergence rates are displayed in Fig. 12. The benefits of VLR-localTS for all Mach numbers and CM-localTS for low Mach number regimes in terms of convergence acceleration and stability of the solution for the Euler equations is clearly demonstrated in these examples. VLR-localTS gives the best convergence rate in all cases. CM-localTS compared to NP-localTS performs better for low Mach numbers. However when $0.3<M<1$, it is seen to give similar convergence rates to the NP-localTS case, and worse convergence rates when $M>1$. This is the expected behavior from what is said in Subsection 4.2. We note that for a Mach number of 0.001, the convergence of the VLR case (Fig. 12a) stalls at around $10^{-11}$. The same phenomena occurs in [1] when they solve the same problem for a Mach of 0.01, they do not solve the Mach 0.001 case. As they indicate, we believe that this happens because of the round-off errors due to the fact that the kinetic energy of the flow is so small compared to the internal energy.

When $M=1.0$ at the inflow, the convergence obtained with the VLR preconditioner is very sensitive to the definition of the reference Mach number $M^{*}$ (Eq. (40)) around the sonic point. This definition depends on the value of the bound parameter $\epsilon_{1}$ that is set to 0.01 in this paper. If $\epsilon_{1}$ is set to 0.1 , the VLR-localTS convergence stalls at approximately $10^{-6}$ (not shown). If it is set to 0.001 , the problem diverges. 

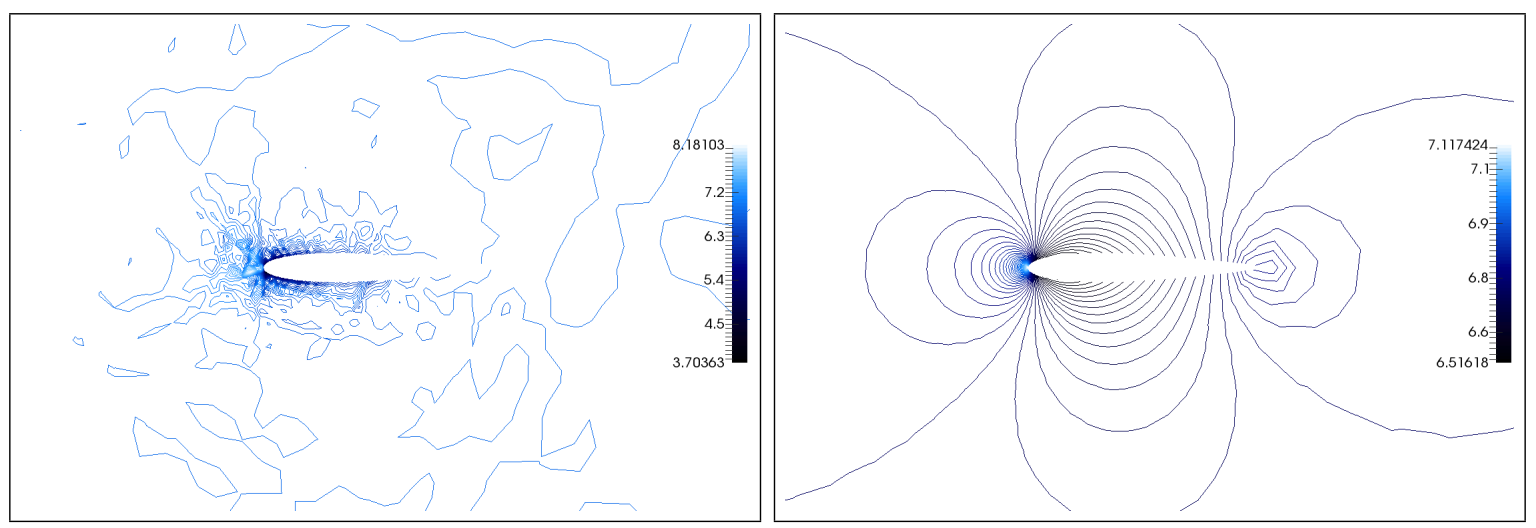

Figure 1: NACA 0012 test case. Pressure contours for an inflow Mach number of 0.001 for the NP (left) and VLR (right) cases with local time step. Due to the very small change in pressure at this Mach number, the contours of $p-p_{\text {ref }}$, that is the change in pressure in relation to a reference, $p_{\text {ref }}=714279$, are plotted.
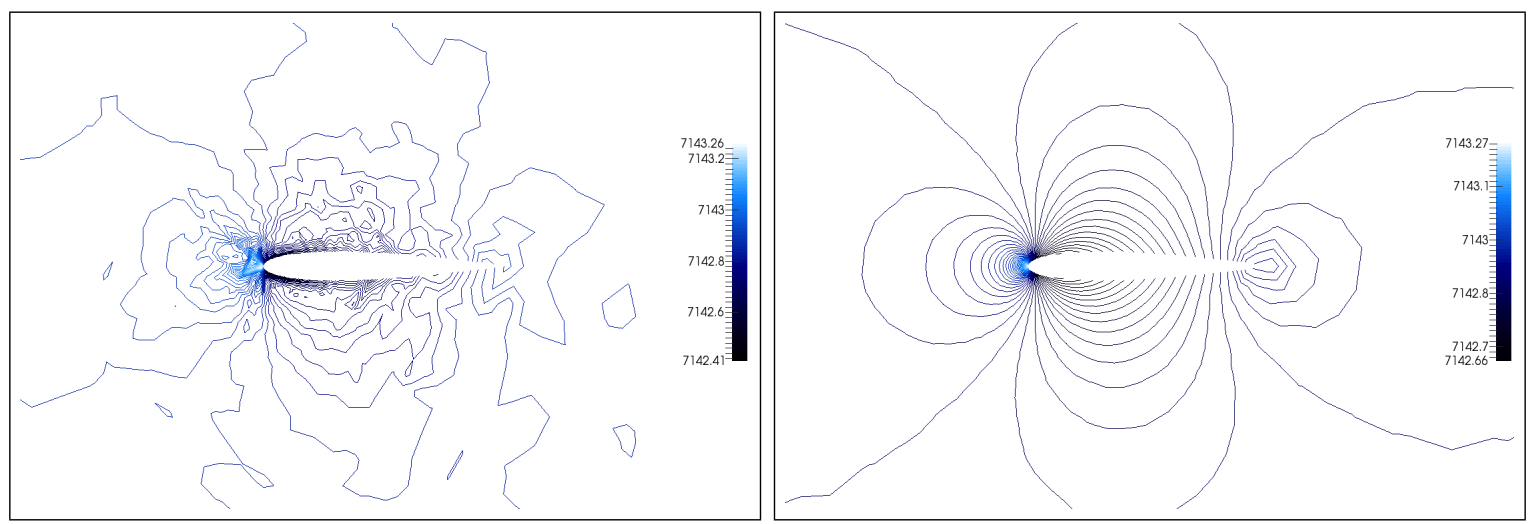

Figure 2: NACA 0012 test case. Pressure contours for an inflow Mach number of 0.01 for the NP (left) and VLR (right) cases with local time step. 



Figure 3: NACA 0012 test case. Pressure contours for an inflow Mach number of 0.1 for the NP (left) and VLR (right) cases with local time step.
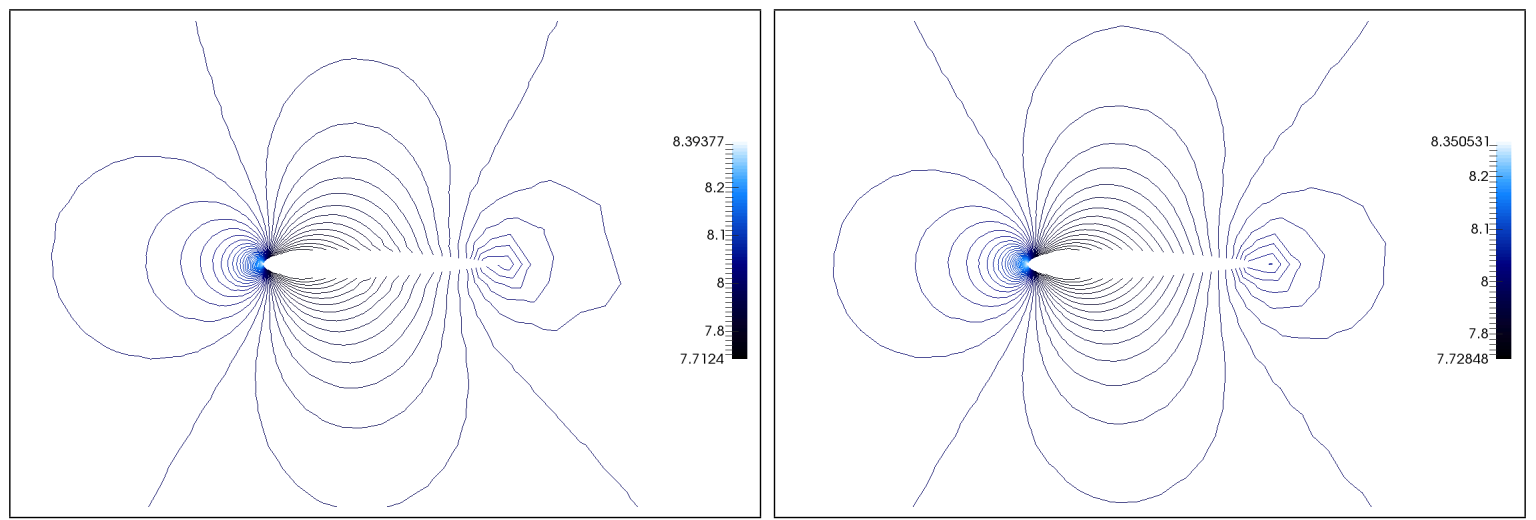

Figure 4: NACA 0012 test case. Pressure contours for an inflow Mach number of 0.3 for the NP (left) and VLR (right) cases with local time step. 

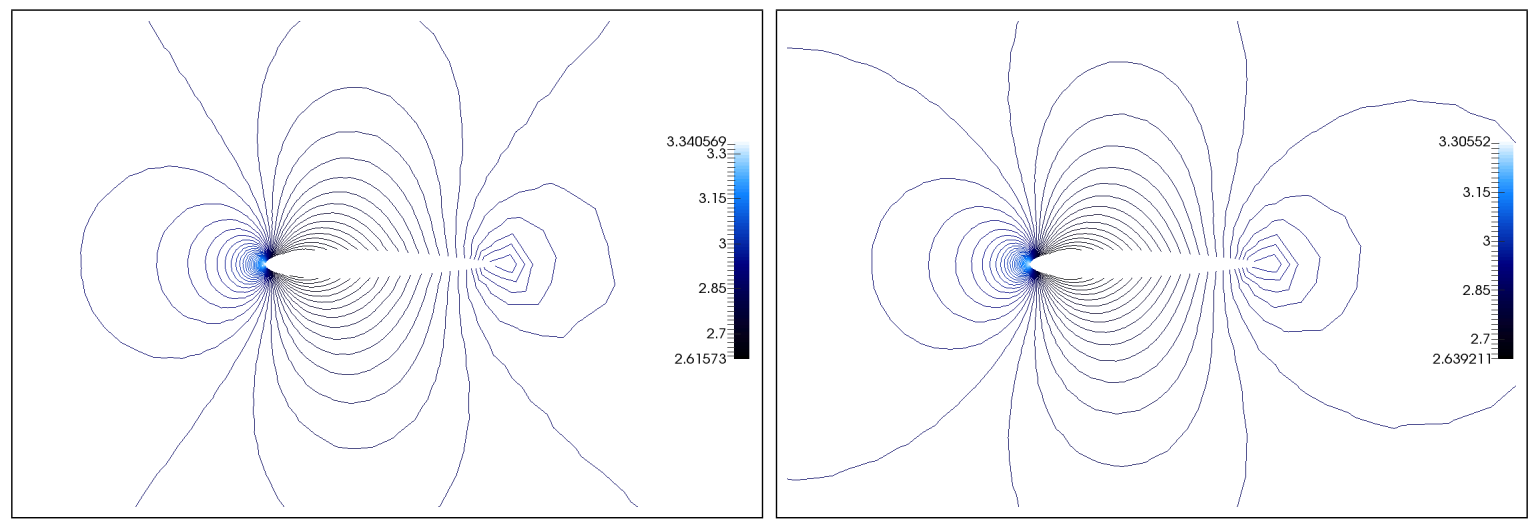

Figure 5: NACA 0012 test case. Pressure contours for an inflow Mach number of 0.5 for the NP (left) and VLR (right) cases with local time step.
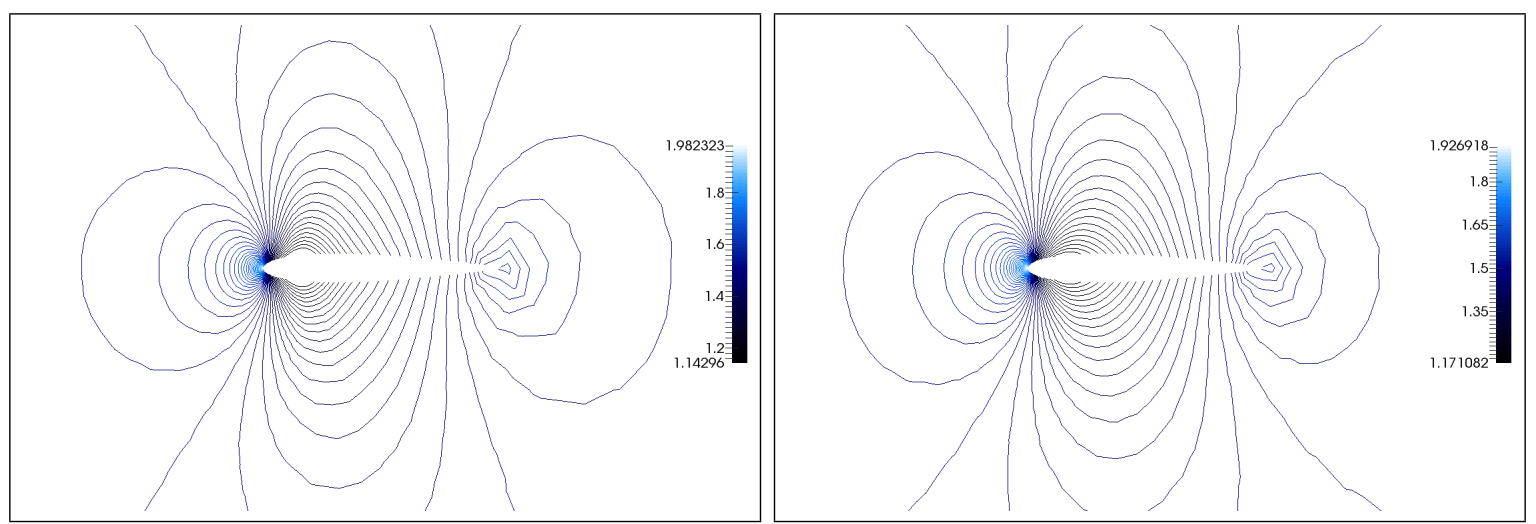

Figure 6: NACA 0012 test case. Pressure contours for an inflow Mach number of 0.7 for the NP (left) and VLR (right) cases with local time step. 

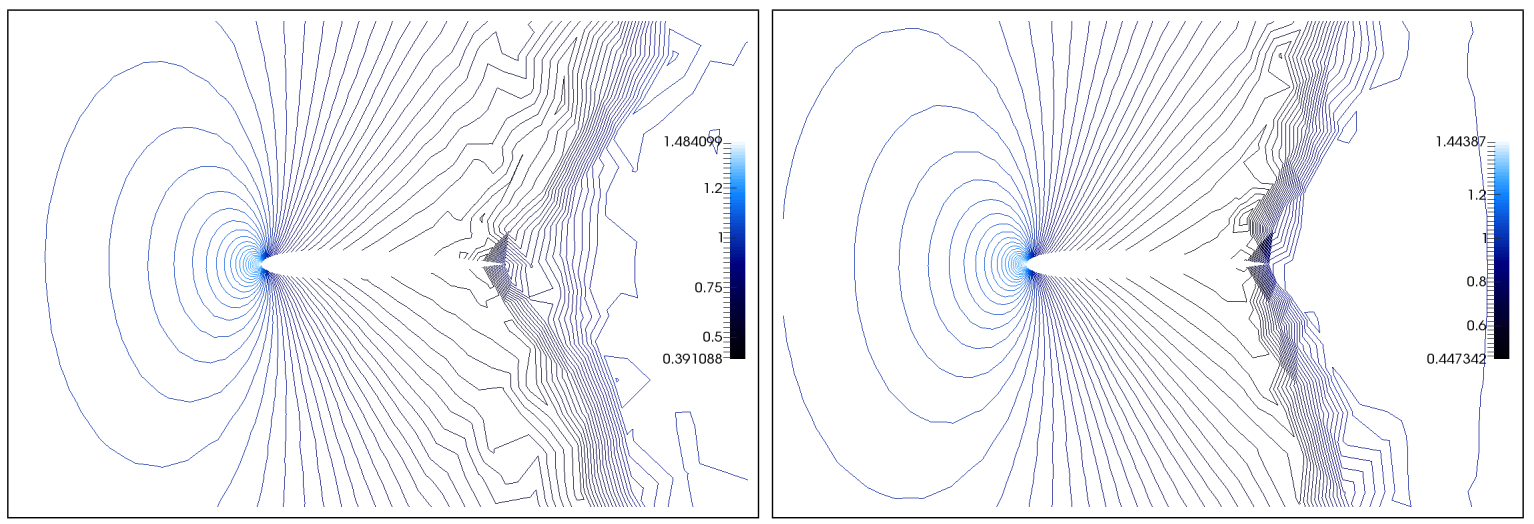

Figure 7: NACA 0012 test case. Pressure contours for an inflow Mach number of 0.9 for the NP (left) and VLR (right) cases with local time step.
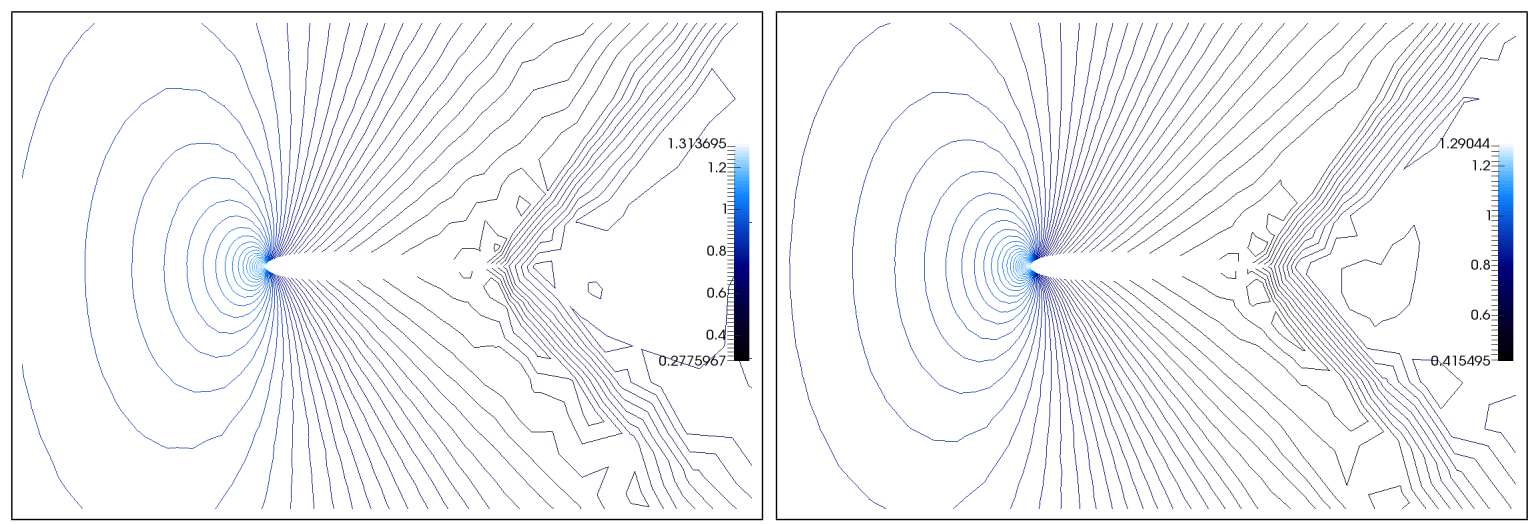

Figure 8: NACA 0012 test case. Pressure contours for an inflow Mach number of 1.0 for the NP (left) and VLR (right) cases with local time step. 

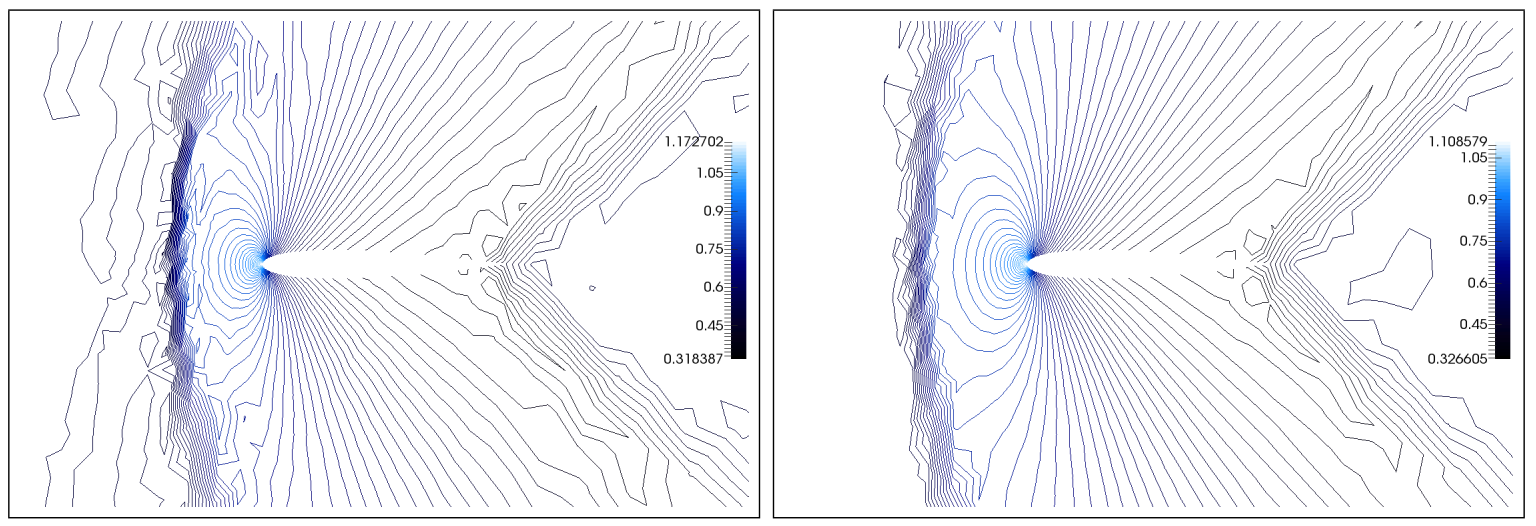

Figure 9: NACA 0012 test case. Pressure contours for an inflow Mach number of 1.2 for the NP (left) and VLR (right) cases with local time step.
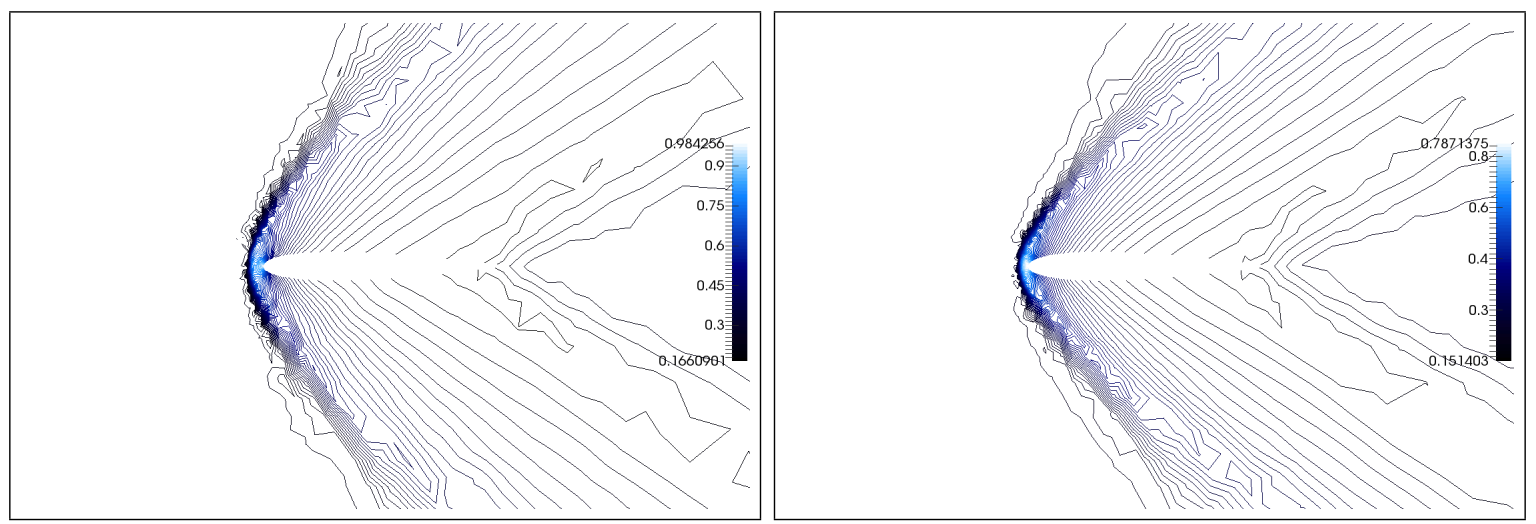

Figure 10: NACA 0012 test case. Pressure contours for an inflow Mach number of 1.8 for the NP (left) and VLR (right) cases with local time step. 

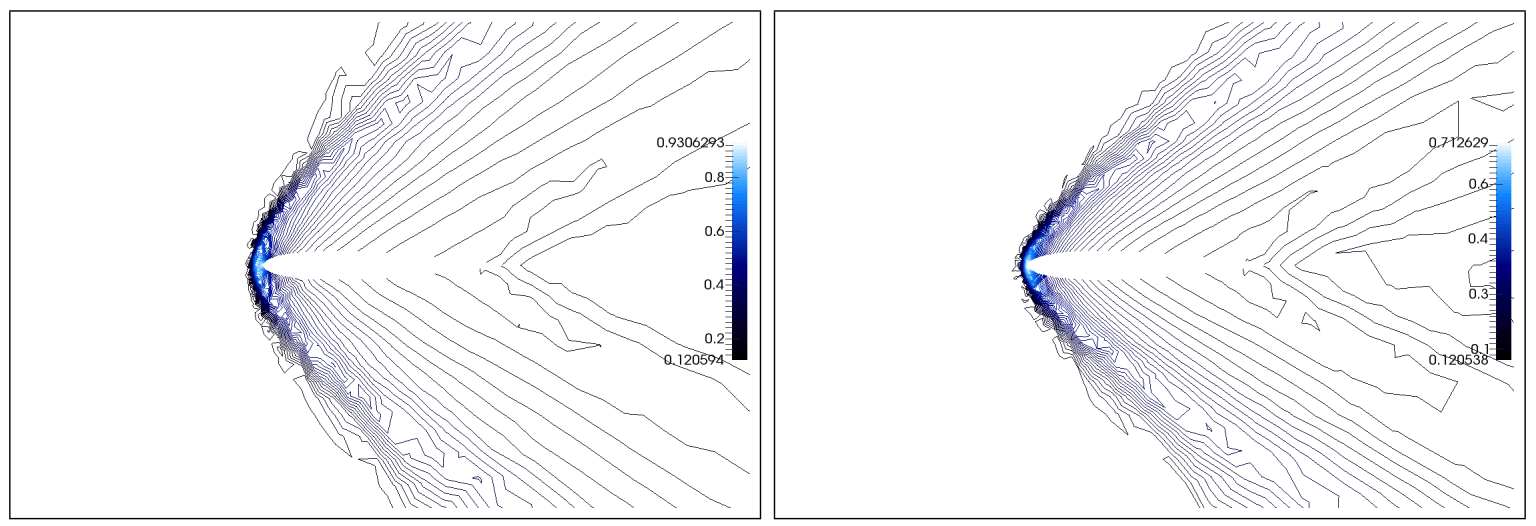

Figure 11: NACA 0012 test case. Pressure contours for an inflow Mach number of 2.0 for the NP (left) and VLR (right) cases with local time step. 




Table 4: NACA 0012 test case. Comparative minimum/maximum Mach numbers over the domain once reached the steady state. The different preconditioning options using global and local time stepping are compared for the NACA0012 test case at different inflow Mach numbers. VLRglobalTS and VLR-localTS values are shown in the same column (VLR) as they give identical results. The dash symbol "_" indicates that the result didn't converge. 


\begin{tabular}{cccccccccccc} 
Mach & 0.001 & 0.01 & 0.1 & 0.3 & 0.5 & 0.7 & 0.9 & 1.0 & 1.2 & 1.8 & 2.0 \\
\hline NP-globalTS & 0.4 & 0.4 & 0.4 & 0.5 & 0.6 & 0.6 & 0.6 & 0.6 & 0.6 & 0.6 & 0.7 \\
\hline NP-localTS & 0.3 & 0.3 & 0.3 & 0.4 & 0.4 & 0.4 & 0.3 & 0.4 & 0.4 & 0.4 & 0.4 \\
\hline VLR-globalTS & 0.5 & 0.5 & 0.5 & 0.5 & 0.6 & 0.6 & 0.6 & 0.6 & 0.6 & 0.8 & 0.7 \\
\hline VLR-localTS & 0.3 & 0.3 & 0.3 & 0.3 & 0.3 & 0.3 & 0.3 & 0.3 & 0.3 & 0.3 & 0.2 \\
\hline CM-globalTS & 0.2 & 0.2 & 0.2 & 0.2 & 0.2 & 0.1 & 0.2 & 0.3 & 0.3 & 0.4 & 0.4 \\
\hline CM-localTS & 0.1 & 0.1 & 0.1 & 0.1 & 0.1 & 0.1 & 0.1 & 0.1 & 0.1 & 0.1 & 0.1 \\
\hline
\end{tabular}

Table 5: NACA 0012 test case. Comparison of the CFL numbers used for the different preconditioning options at different inflow Mach numbers. 
(a) $M=0.001$

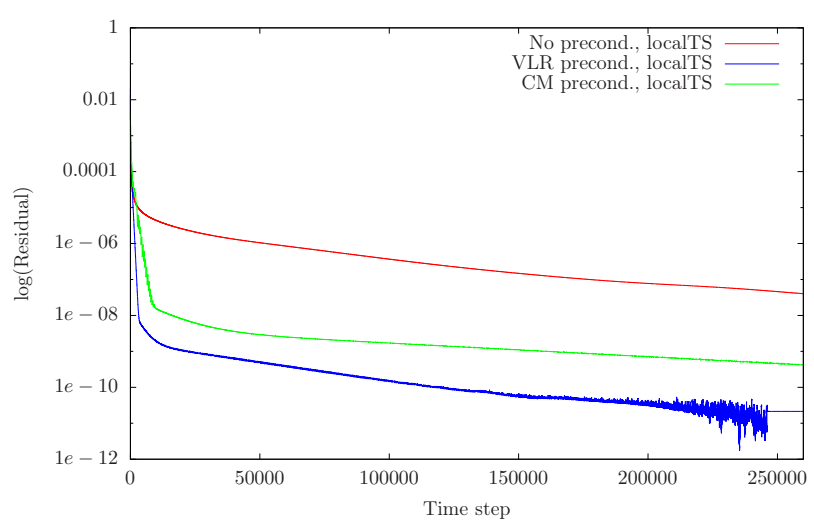

(c) $M=0.1$

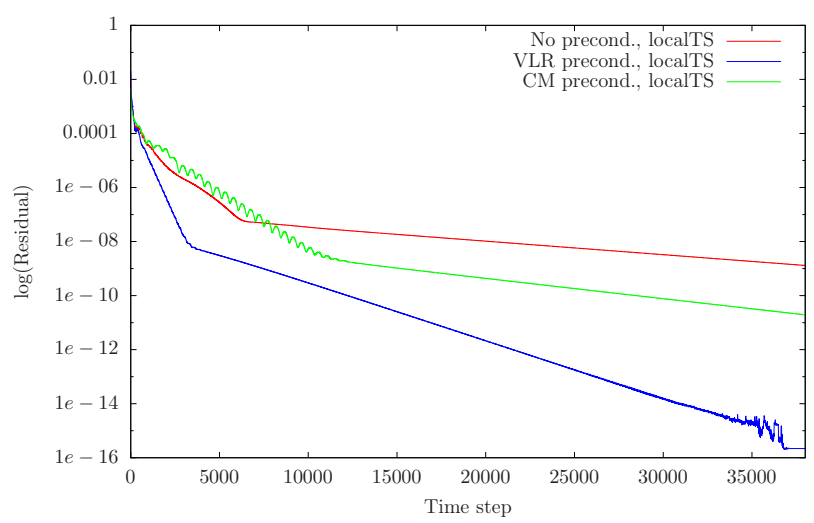

(e) $M=0.5$

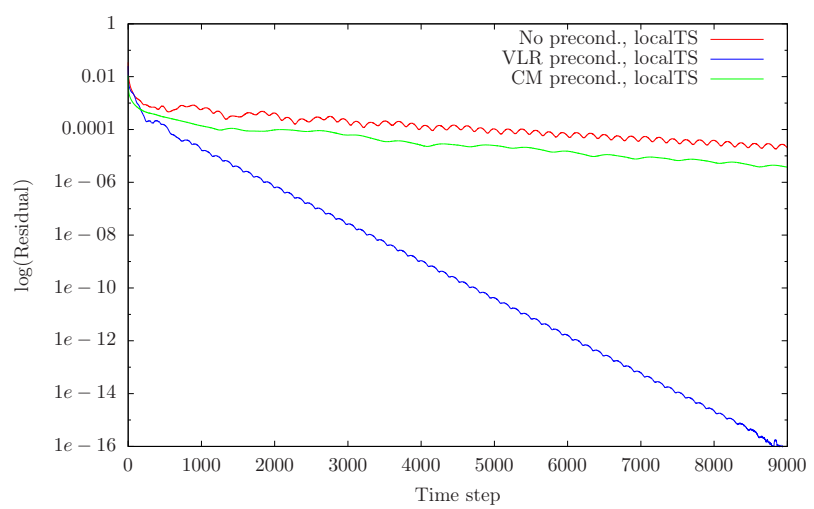

(b) $M=0.01$



(d) $M=0.3$

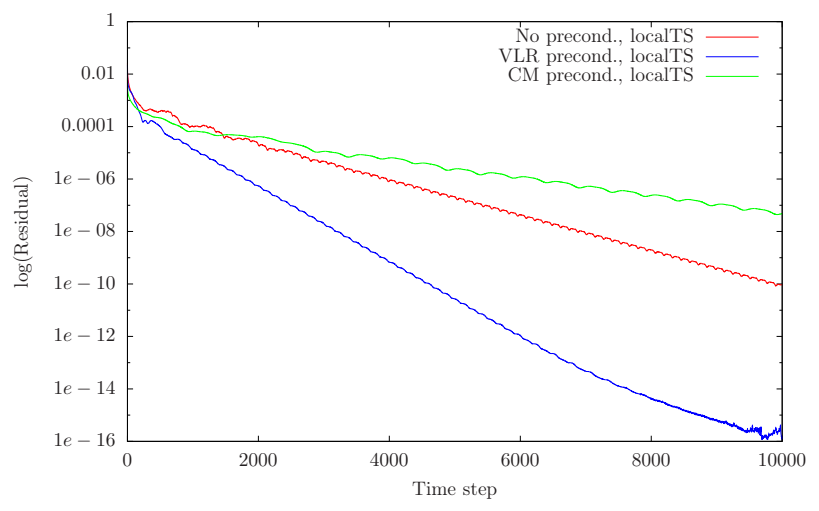

(f) $M=0.7$

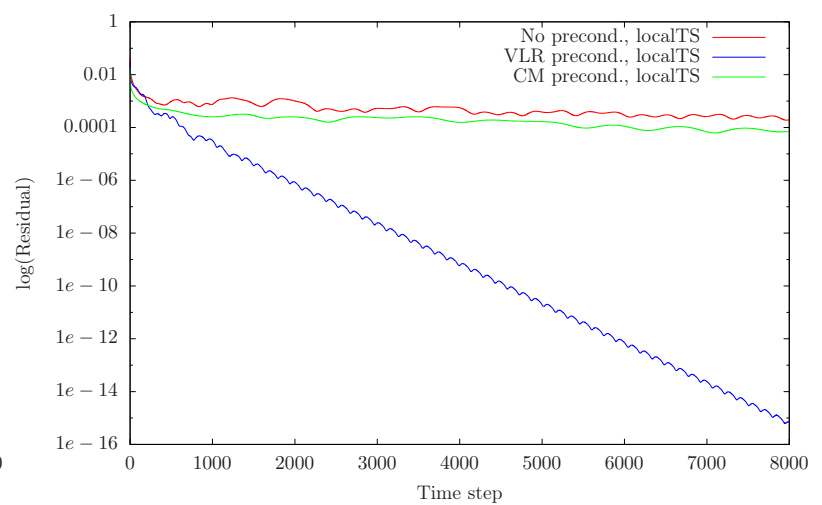


(g) $M=0.9$

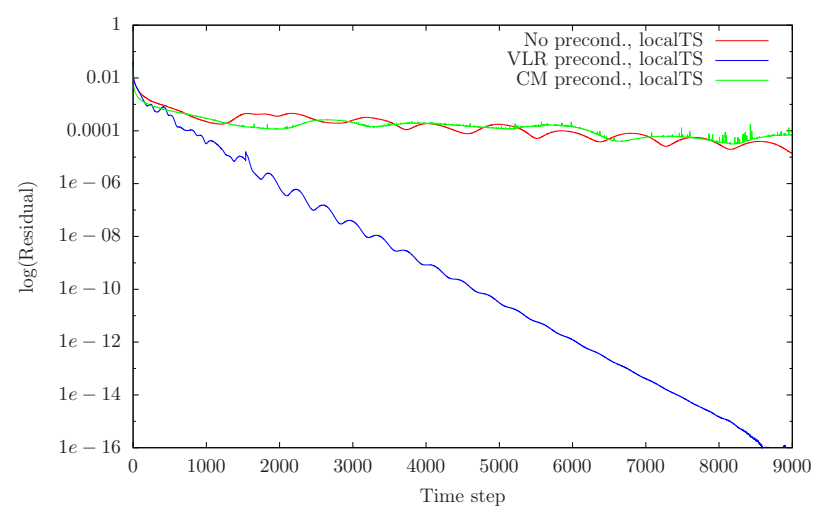

(i) $M=1.2$

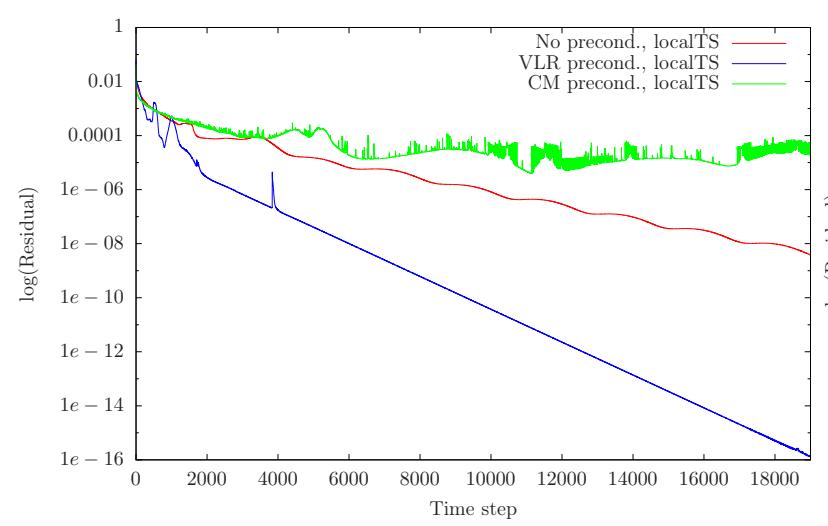

(k) $M=2.0$

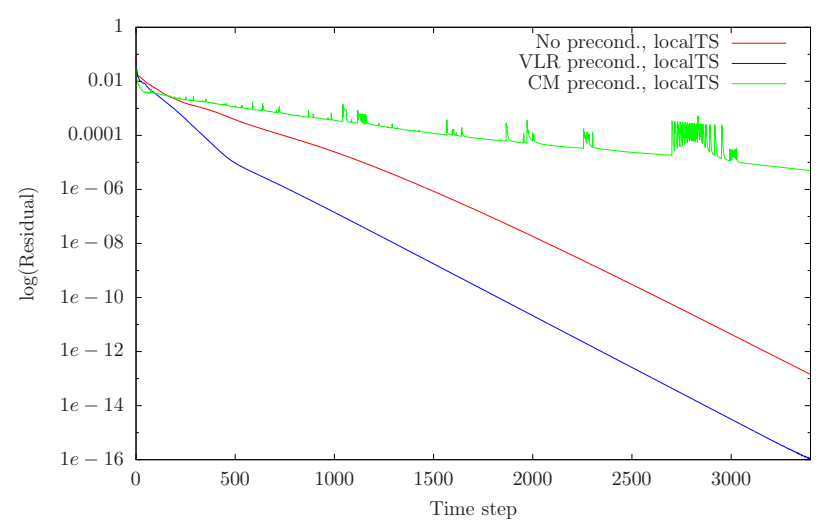

(h) $M=1.0$

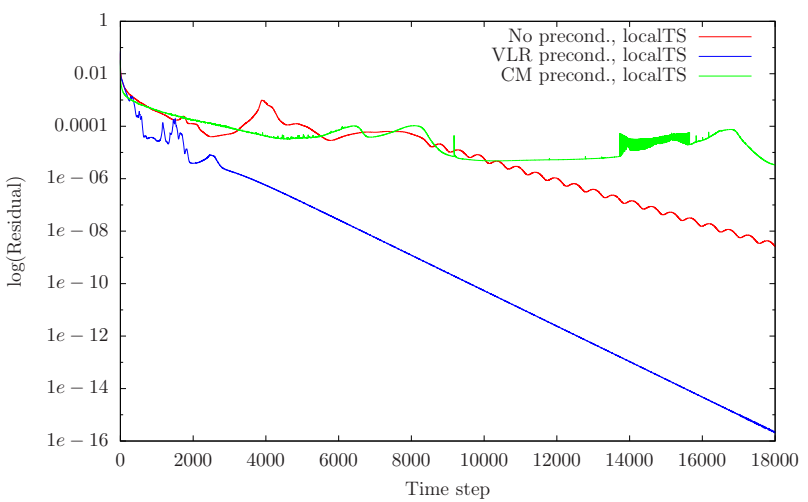

(j) $M=1.8$

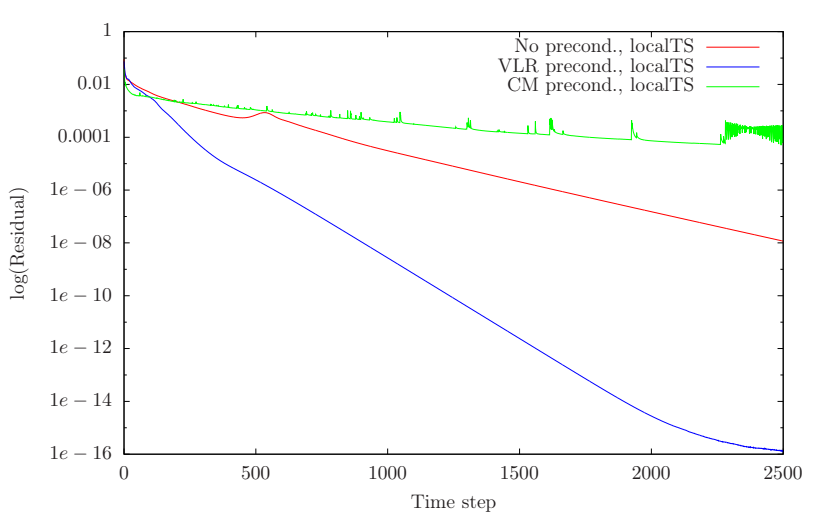

Figure 12: NACA 0012 test case. Residual-convergence rates over the time step using the three preconditioning options (NP, VLR, and CM) with local time at different inflow Mach numbers. We use the residual defined by equation (68). 


\subsection{ONERA M6 wing}

The ONERA M6 wing configuration details and experimental results are found in [53]. The results presented here correspond to inviscid transonic flow at a Mach number of 0.8395 and an angle of attack of $3.06^{\circ}$. As in the NACA 0012 test case, the rest of the initial conditions are $\rho=1$, $\|\mathbf{u}\|=1, T=1$, and $c_{\mathrm{p}}$ and $c_{\mathrm{v}}$ are determined to obtain the desired inflow Mach numbers. An unstructured tetrahedral mesh is used on this simulation consisting of 472026 elements and 94481 grid points. The VLR and NP options are tested for this case. The CM option only improving performance for low Mach number regimes is not considered in this problem. The NP-localTS option does not converge for this problem.

Convergence for the NP-globalTS and VLR-localTS cases are compared in Fig. 13. We observe that the VLR-localTS configuration considerably accelerates the convergence compared to the NP-globalTS one.

The pressure contours on the upper and lower surface of the wing for the VLR-localTS case with $C F L=0.2$ are shown in Fig. 14. In the upper surface contours we can identify the complex sharp shock structure that is formed.

The pressure coefficient values of the VLR-localTS simulation at seven spanwise orthogonal sections of the wing are compared to the experimental results in [53] in Fig. 15. The seven sections are at $20 \%(y=0.23926 m), 44 \%(y=0.526372 m), 65 \%(y=0.777595 m), 80 \%(y=$ $0.95704 m), 90 \%(y=1.07667 m), 95 \%(y=1.136485 m)$, and $99 \%(y=1.184337 m)$ of the semispan that goes from $y=0$ to $y=1.1963 \mathrm{~m}$, where $y$ is the span-wise coordinate. We observe some differences between the results and the experimental data near the shock located at $x=0.6$ on the horizontal axis for all cases, whether preconditioned or not. Considering that we are comparing the experimental results with an Euler solution, the obtained pressure coefficient values at the different sections are reasonable (see for instance [54]). We also observe a difference near the trailing edge when comparing our results with the experiments. We believe that this difference could be due to several reasons: the way we impose boundary conditions, the lack of physical viscosity, the fact that there is no local mesh refinement at the trailing edge, etc. 


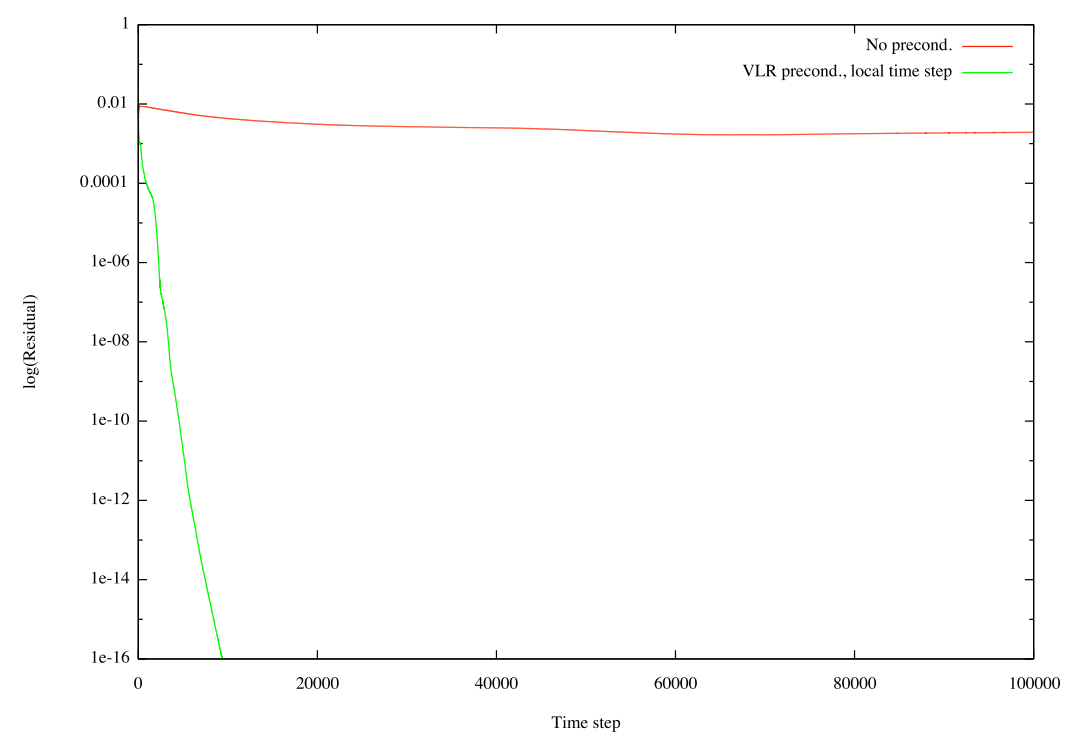

Figure 13: ONERA M6 test case. Convergence rate comparison between the non preconditioned case using a global time stepping (red line) and the VLR preconditioned case using a local time stepping (green line). We use the residual defined by equation (68).
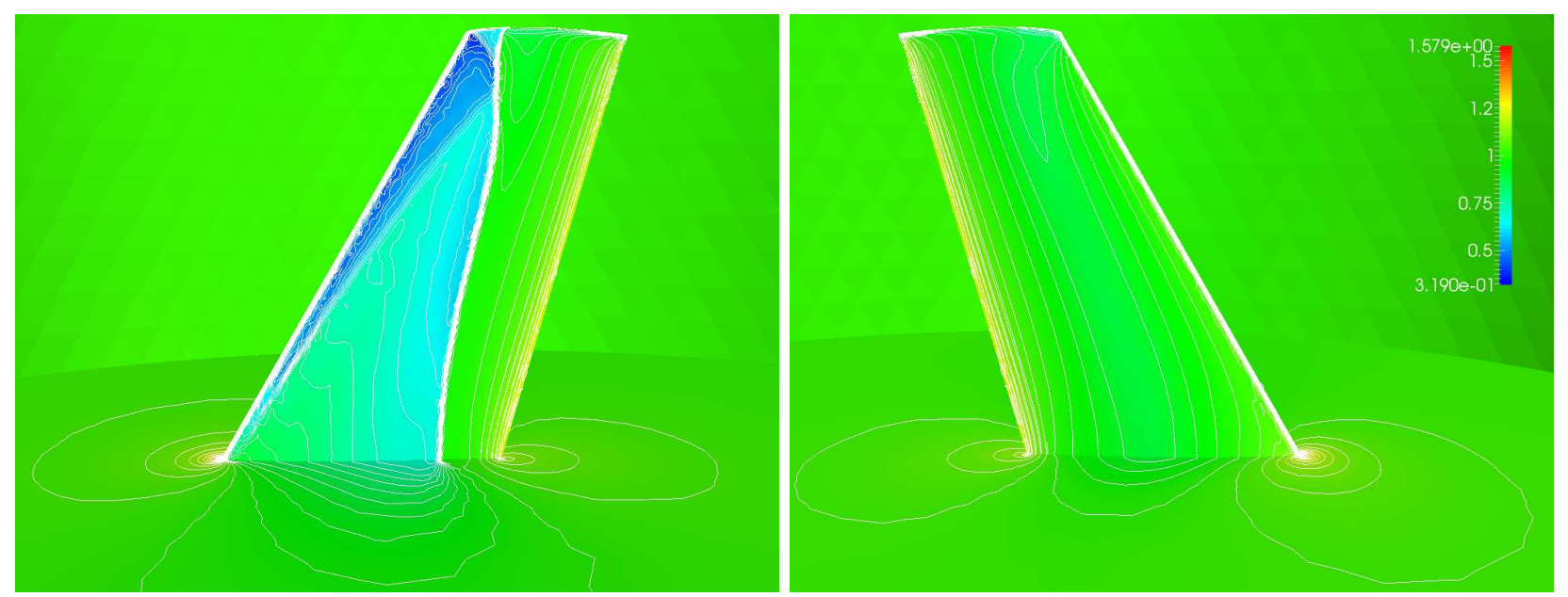

Figure 14: ONERA M6 test case. Pressure contours on the upper (left figure) and lower (right figure) surfaces for the VLR preconditioned case with local time step. 
(a) $20 \%$ of the semi-span

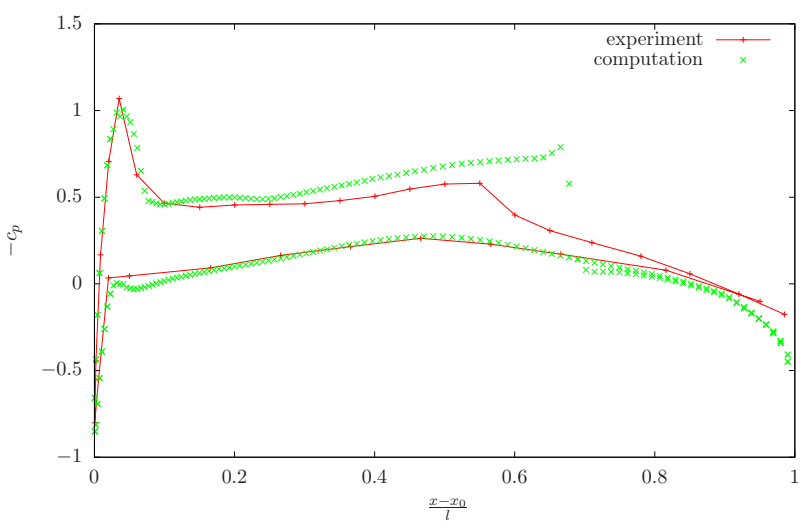

(c) $65 \%$ of the semi-span

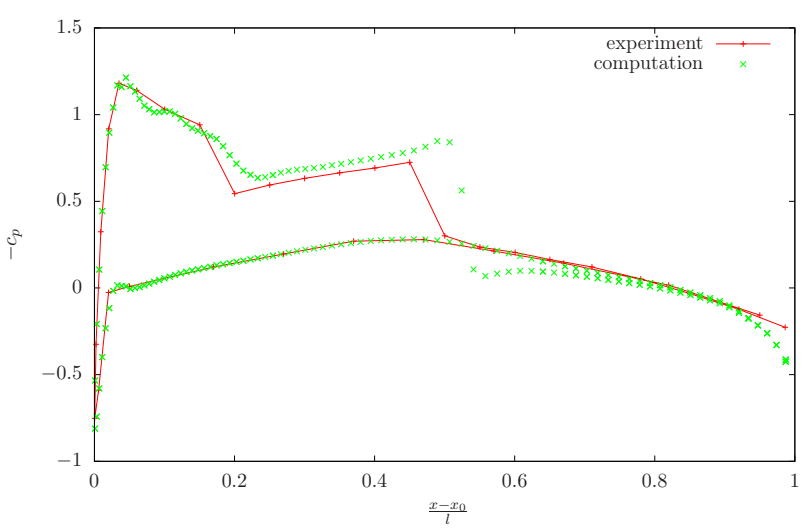

(e) $90 \%$ of the semi-span

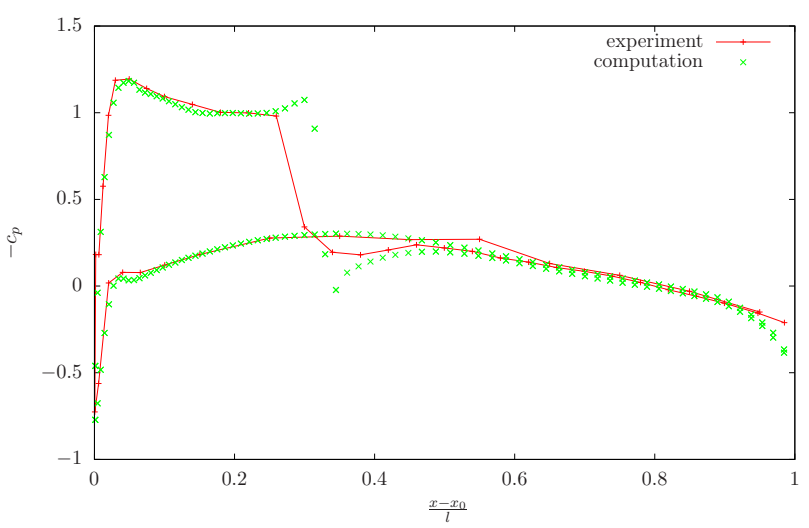

(b) $44 \%$ of the semi-span

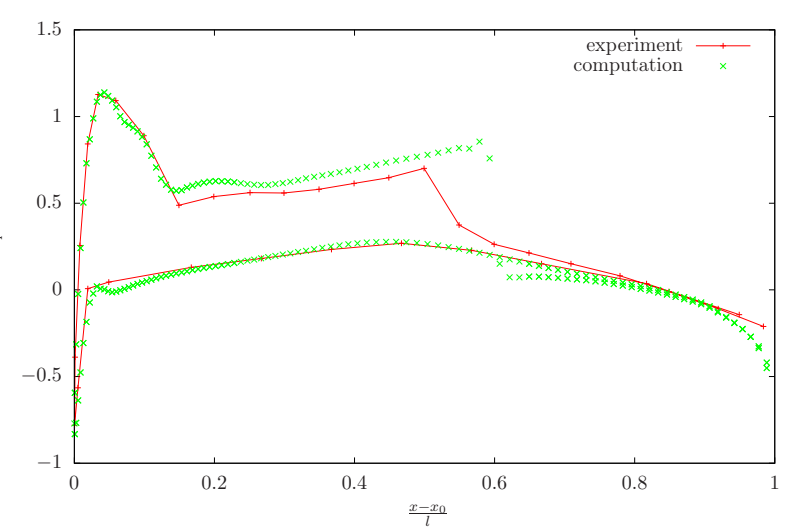

(d) $80 \%$ of the semi-span

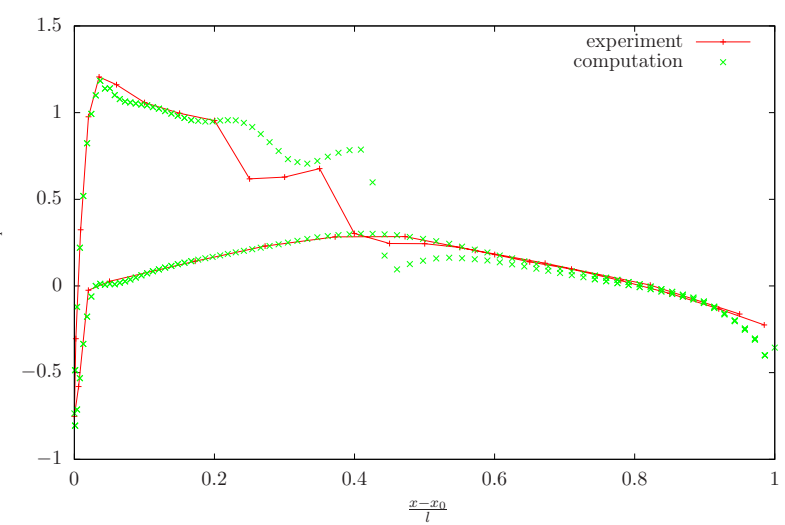

(f) $95 \%$ of the semi-span

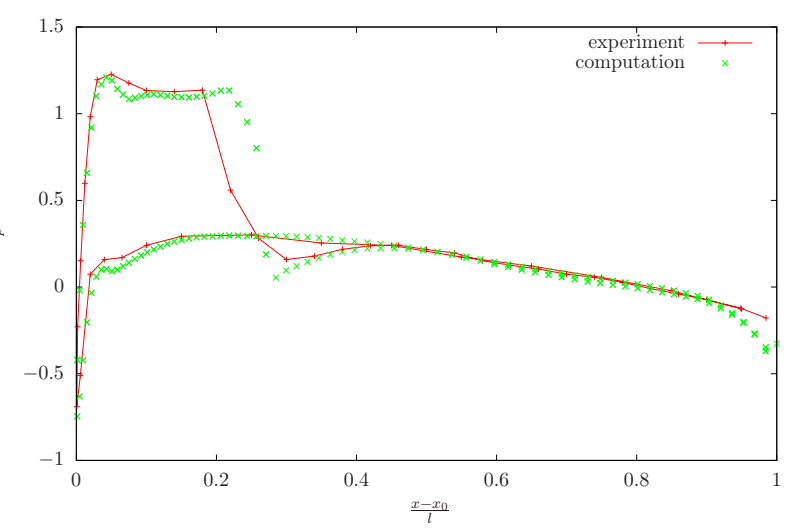


(g) $99 \%$ of the semi-span

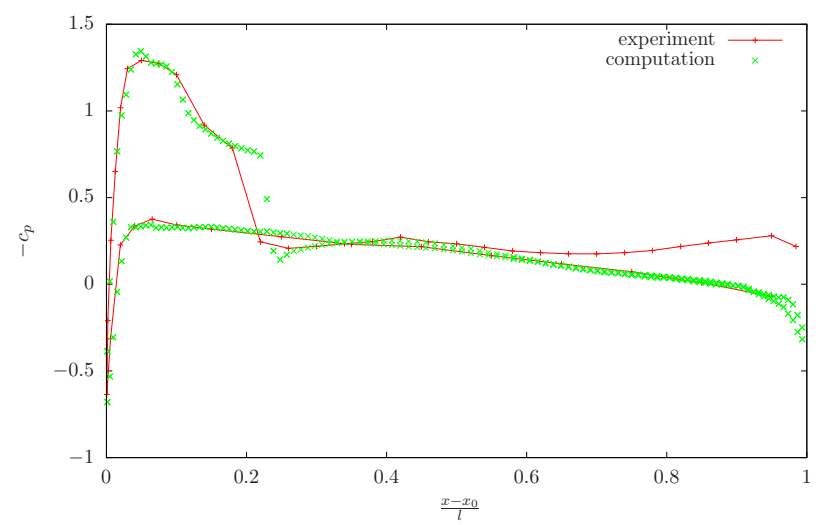

Figure 15: ONERA M6 test case. The $c_{p}$ values for the experimental and VLR-localTS's results are compared on seven span-wise sections of the wing.

\section{Conclusions}

In the present paper a variational multiscale stabilization for the locally preconditioned Euler equations is introduced. This stabilization method, that we call P-VMS, is applied to the finite element discretization of steady compressible flows. Time discretization is approached by a linear explicit scheme in order to present and assess P-VMS in the most simple and direct way possible. The local and global time stepping are compared.

Due to the effect of local preconditioning on the hyperbolic equations, the preconditioned system is better suited for the computation of the VMS for a wide-range of Mach numbers. The combined action of local preconditioning and VMS results in a robust and precise numerical method. As local preconditioning is applied before any discretization is done, it does not necessarily add extra computational cost compared to the non-preconditioned option. Indeed, the product $\mathbf{P A}^{i}$ can be computed analytically and the corresponding expression introduced into the code.

VLR and CM preconditioners are tested over two benchmarks comprising an ample range of Mach numbers. We observe that preconditioning gives improvement in terms of convergence acceleration, stability, and smoothness of the solution even for very low Mach numbers. The use of a local time stepping compared to a global one, accelerates the convergence to the steady solution as well. VLR preconditioning gives better improvement than CM one on the convergence rate and 
applies from subsonic to supersonic regimes. CM preconditioning improves the convergence for low Mach number flows. 


\section{Appendix A. Jacobian matrices}

$$
\begin{aligned}
& \mathbf{A}^{1}(\boldsymbol{\Phi})=\left(\begin{array}{ccccc}
\left(2-\frac{R}{c_{\mathrm{v}}}\right) \frac{U_{1}}{\rho} & -\frac{R}{c_{\mathrm{v}}} \frac{U_{2}}{\rho} & -\frac{R}{c_{\mathrm{v}}} \frac{U_{3}}{\rho} & -\frac{U_{1}^{2}}{\rho^{2}}+\frac{1}{2} \frac{R}{c_{\mathrm{v}}} \frac{U_{k} U_{k}}{\rho^{2}} & \frac{R}{c_{\mathrm{v}}} \\
\frac{U_{2}}{\rho} & \frac{U_{1}}{\rho} & 0 & -\frac{U_{1} U_{2}}{\rho^{2}} & 0 \\
\frac{U_{3}}{\rho} & 0 & \frac{U_{1}}{\rho} & -\frac{U_{1} U_{3}}{\rho^{2}} & 0 \\
1 & 0 & 0 & 0 & 0 \\
\frac{1}{\rho}\left(E+p-\frac{R}{c_{\mathrm{v}}} \frac{U_{1}^{2}}{\rho}\right) & \frac{-R}{c_{\mathrm{v}}} \frac{U_{1} U_{2}}{\rho^{2}} & \frac{-R}{c_{\mathrm{v}}} \frac{U_{1} U_{3}}{\rho^{2}} & \frac{-U_{1}}{\rho^{2}}\left(E+p-\frac{1}{2} \frac{R}{c_{\mathrm{v}}} \frac{U_{k} U_{k}}{\rho}\right) & \left(1+\frac{R}{c_{\mathrm{v}}}\right) \frac{U_{1}}{\rho}
\end{array}\right) \\
& \mathbf{A}^{2}(\boldsymbol{\Phi})=\left(\begin{array}{ccccc}
\frac{U_{2}}{\rho} & \frac{U_{1}}{\rho} & 0 & -\frac{U_{2} U_{1}}{\rho^{2}} & 0 \\
-\frac{R}{c_{\mathrm{v}}} \frac{U_{1}}{\rho} & \left(2-\frac{R}{c_{\mathrm{v}}}\right) \frac{U_{2}}{\rho} & -\frac{R}{c_{\mathrm{v}}} \frac{U_{3}}{\rho} & -\frac{U_{2}^{2}}{\rho^{2}}+\frac{1}{2} \frac{R}{c_{\mathrm{v}}} \frac{U_{k} U_{k}}{\rho^{2}} & \frac{R}{c_{\mathrm{v}}} \\
0 & \frac{U_{3}}{\rho} & \frac{U_{2}}{\rho} & -\frac{U_{2} U_{3}}{\rho^{2}} & 0 \\
0 & 1 & 0 & 0 & 0 \\
\frac{-R}{c_{\mathrm{v}}} \frac{U_{2} U_{1}}{\rho^{2}} & \frac{1}{\rho}\left(E+p-\frac{R}{c_{\mathrm{v}}} \frac{U_{2}^{2}}{\rho}\right) & \frac{-R}{c_{\mathrm{v}}} \frac{U_{2} U_{3}}{\rho^{2}} & \frac{-U_{2}}{\rho^{2}}\left(E+p-\frac{1}{2} \frac{R}{c_{\mathrm{v}}} \frac{U_{k} U_{k}}{\rho}\right) & \left(1+\frac{R}{c_{\mathrm{v}}}\right) \frac{U_{2}}{\rho} \\
\frac{U_{3}}{\rho} & 0 & \frac{U_{1}}{\rho} & -\frac{U_{3} U_{1}}{\rho^{2}} & 0 \\
0 & \frac{U_{3}}{\rho} & \frac{U_{2}}{\rho} & -\frac{U_{3} U_{2}}{\rho^{2}} & \frac{R}{c_{\mathrm{v}}} \\
-\frac{R}{c_{\mathrm{v}}} \frac{U_{1}}{\rho} & -\frac{R}{c_{\mathrm{v}}} \frac{U_{2}}{\rho} & \left(2-\frac{R}{c_{\mathrm{v}}}\right) \frac{U_{3}}{\rho} & -\frac{U_{3}^{2}}{\rho^{2}}+\frac{1}{2} \frac{R}{c_{\mathrm{v}}} \frac{U_{k}}{\rho^{2}} & 0 \\
0 & 0 & 1 & 0 & \left(1+\frac{R}{c_{\mathrm{v}}} \frac{U_{3}}{\rho}\right.
\end{array}\right) \\
& \mathbf{A}^{3}(\boldsymbol{\Phi})=
\end{aligned}
$$

\section{Appendix B. Euler equations in symmetrizing variables}

We introduce the symmetrization of the Euler equations proposed by [55]. The symmetrizing variables are

$$
\mathrm{d} \boldsymbol{\Phi}_{\mathrm{s}}=\left(\begin{array}{lllll}
\mathrm{d} u_{1} & \mathrm{~d} u_{2} & \mathrm{~d} u_{3} & \frac{\mathrm{d} p}{\rho c} & \mathrm{~d} p-c^{2} \mathrm{~d} \rho
\end{array}\right)^{\mathrm{T}},
$$

where $\mathrm{d} p-c^{2} \mathrm{~d} \rho=\frac{p}{c_{\mathrm{v}}} \mathrm{d} s$. They are related to the conservative variables through the expression $\mathrm{d} \boldsymbol{\Phi}_{\mathrm{s}}=\frac{\partial \boldsymbol{\Phi}_{\mathrm{s}}}{\partial \boldsymbol{\Phi}} \mathrm{d} \boldsymbol{\Phi}$, where

$$
\frac{\partial \Phi_{\mathrm{s}}}{\partial \Phi}=\left(\begin{array}{ccccc}
\frac{1}{\rho} & 0 & 0 & -\frac{u_{1}}{\rho} & 0 \\
0 & \frac{1}{\rho} & 0 & -\frac{u_{2}}{\rho} & 0 \\
0 & 0 & \frac{1}{\rho} & -\frac{u_{3}}{\rho} & 0 \\
-\frac{R}{c_{\mathrm{v}}} \frac{u_{1}}{\rho c} & -\frac{R}{c_{\mathrm{v}}} \frac{u_{2}}{\rho c} & -\frac{R}{c_{\mathrm{v}}} \frac{u_{3}}{\rho c} & \frac{1}{2} \frac{R}{c_{\mathrm{v}}} \frac{u_{k} u_{k}}{\rho c} & \frac{R}{c_{\mathrm{v}}} \frac{1}{\rho c} \\
-\frac{R}{c_{\mathrm{v}}} u_{1} & -\frac{R}{c_{\mathrm{v}}} u_{2} & -\frac{R}{c_{\mathrm{v}}} u_{3} & \frac{1}{2} \frac{R}{c_{\mathrm{v}}} u_{k} u_{k}-c^{2} & \frac{R}{c_{\mathrm{v}}}
\end{array}\right),
$$


and its inverse

$$
\frac{\partial \Phi}{\partial \boldsymbol{\Phi}_{\mathrm{s}}}=\left(\begin{array}{ccccc}
\rho & 0 & 0 & \frac{\rho u_{1}}{c} & -\frac{u_{1}}{c^{2}} \\
0 & \rho & 0 & \frac{\rho u_{2}}{c} & -\frac{u_{2}}{c^{2}} \\
0 & 0 & \rho & \frac{\rho u_{3}}{c} & -\frac{u_{3}}{c^{2}} \\
0 & 0 & 0 & \frac{\rho}{c} & -\frac{1}{c^{2}} \\
\rho u_{1} & \rho u_{2} & \rho u_{3} & \frac{c_{v}}{R} \rho c+\frac{1}{2} \frac{\rho u_{k} u_{k}}{c} & -\frac{1}{2} \frac{u_{k} u_{k}}{c^{2}}
\end{array}\right) .
$$

we then obtain the symmetric jacobian matrices in the symmetrizing variables, $\mathbf{A}_{\mathrm{s}}^{i}=\frac{\partial \boldsymbol{\Phi}_{\mathrm{s}}}{\partial \boldsymbol{\Phi}} \mathbf{A}^{i} \frac{\partial \Phi}{\partial \boldsymbol{\Phi}_{\mathrm{s}}}$, $i=1, \ldots, 3$, which are explicitly written as:

$\mathbf{A}_{\mathrm{s}}^{1}=\left(\begin{array}{ccccc}u_{1} & 0 & 0 & c & 0 \\ 0 & u_{1} & 0 & 0 & 0 \\ 0 & 0 & u_{1} & 0 & 0 \\ c & 0 & 0 & u_{1} & 0 \\ 0 & 0 & 0 & 0 & u_{1}\end{array}\right), \mathbf{A}_{\mathrm{s}}^{2}=\left(\begin{array}{ccccc}u_{2} & 0 & 0 & 0 & 0 \\ 0 & u_{2} & 0 & c & 0 \\ 0 & 0 & u_{2} & 0 & 0 \\ 0 & c & 0 & u_{2} & 0 \\ 0 & 0 & 0 & 0 & u_{2}\end{array}\right), \mathbf{A}_{\mathrm{s}}^{3}=\left(\begin{array}{ccccc}u_{3} & 0 & 0 & 0 & 0 \\ 0 & u_{3} & 0 & 0 & 0 \\ 0 & 0 & u_{3} & c & 0 \\ 0 & 0 & c & u_{3} & 0 \\ 0 & 0 & 0 & 0 & u_{3}\end{array}\right)$.

Then, (4) is equivalent to the symmetric system

$$
\frac{\partial \boldsymbol{\Phi}_{\mathrm{s}}}{\partial t}+\mathbf{A}_{\mathrm{s}}^{i} \frac{\partial \boldsymbol{\Phi}_{\mathrm{s}}}{\partial x_{i}}=0
$$

\section{Appendix C. Euler equations in symmetrizing variables and streamline coordinates}

System (B.5) can be simplified even more by rotating the reference coordinates, $\mathbf{x}=\left(x_{1}, x_{2}, x_{3}\right)$, to obtain a coordinate system $\xi=\left(\xi_{1}, \xi_{2}, \xi_{3}\right)$, in which the flow is in the positive $\xi_{1}$-direction. They are called streamline coordinates. We write $\xi=\mathbf{R} \mathbf{x}$, where $\mathbf{R}=\mathbf{R}_{x_{2}} \mathbf{R}_{x_{3}}$,

$$
\mathbf{R}_{x_{2}}=\left(\begin{array}{ccc}
\cos \alpha & 0 & \sin \alpha \\
0 & 1 & 0 \\
-\sin \alpha & 0 & \cos \alpha
\end{array}\right), \quad \mathbf{R}_{x_{3}}=\left(\begin{array}{ccc}
\cos \theta & \sin \theta & 0 \\
-\sin \theta & \cos \theta & 0 \\
0 & 0 & 1
\end{array}\right)
$$

$\mathbf{R}_{x_{2}}$ rotates the $x_{1} x_{3}$-plane counter-clockwise (when the $x_{2}$-axis points toward the observer, and the coordinate system is right-handed), and $\mathbf{R}_{x_{3}}$ rotates the $x_{1} x_{2}$-plane clockwise (when the $x_{3}$-axis 
points toward the observer, and the coordinate system is right-handed). The product reads:

$$
\mathbf{R}=\left(\begin{array}{ccc}
\cos \alpha \cos \theta & \cos \alpha \sin \theta & \sin \alpha \\
-\sin \theta & \cos \theta & 0 \\
-\sin \alpha \cos \theta & -\sin \alpha \sin \theta & \cos \alpha
\end{array}\right)
$$

For the flow to be in the positive $\xi_{1}$-direction, the angles $\theta$ and $\alpha$ or the velocity should satisfy

$$
\left\{\begin{array}{l}
\sin \theta=\frac{u_{2}}{\sqrt{u_{1}^{2}+u_{2}^{2}}} \\
\cos \theta=\frac{u_{1}}{\sqrt{u_{1}^{2}+u_{2}^{2}}}
\end{array}, \quad\left\{\begin{array}{rl}
\sin \alpha=\frac{u_{3}}{\|\mathbf{u}\|} \\
\cos \alpha=\frac{\sqrt{u_{1}^{2}+u_{2}^{2}}}{\|\mathbf{u}\|}
\end{array}, \quad\left\{\begin{array}{l}
u_{1}=\|\mathbf{u}\| \cos \alpha \cos \theta \\
u_{2}=\|\mathbf{u}\| \cos \alpha \sin \theta \\
u_{3}=\|\mathbf{u}\| \sin \alpha .
\end{array}\right.\right.\right.
$$

The velocity in the streamline coordinates is $\mathbf{q}=R \mathbf{u}$, then $q_{1}=\|\mathbf{u}\|$ and $q_{2}=q_{3}=0$. Therefore the symmetrizing variables with streamwise coordinates are

$$
\mathrm{d} \boldsymbol{\Phi}_{\mathrm{ss}}=\left(\begin{array}{lllll}
\mathrm{d} q_{1} & \mathrm{~d} q_{2} & \mathrm{~d} q_{3} & \frac{\mathrm{d} p}{\rho c} & \mathrm{~d} p-c^{2} \mathrm{~d} \rho
\end{array}\right)^{\mathrm{T}} .
$$

They are expressed in terms of the symmetrizing and conservative variables as d $\boldsymbol{\Phi}_{\mathrm{ss}}=\frac{\partial \boldsymbol{\Phi}_{\mathrm{ss}}}{\partial \boldsymbol{\Phi}_{\mathrm{s}}} \mathrm{d} \boldsymbol{\Phi}_{\mathrm{s}}$ and $\mathrm{d} \boldsymbol{\Phi}_{\mathrm{ss}}=\frac{\partial \boldsymbol{\Phi}_{\mathrm{ss}}}{\partial \boldsymbol{\Phi}} \mathrm{d} \boldsymbol{\Phi}$, respectively, where

$$
\frac{\partial \boldsymbol{\Phi}_{\mathrm{ss}}}{\partial \boldsymbol{\Phi}_{\mathrm{s}}}=\left(\begin{array}{ccccc}
\cos \alpha \cos \theta & \cos \alpha \sin \theta & \sin \alpha & 0 & 0 \\
-\sin \theta & \cos \theta & 0 & 0 & 0 \\
-\sin \alpha \cos \theta & -\sin \alpha \sin \theta & \cos \alpha & 0 & 0 \\
0 & 0 & 0 & 1 & 0 \\
0 & 0 & 0 & 0 & 1
\end{array}\right)
$$

its inverse is

$$
\frac{\partial \Phi_{\mathrm{s}}}{\partial \Phi_{\mathrm{ss}}}=\left(\begin{array}{ccccc}
\cos \alpha \cos \theta & -\sin \theta & -\sin \alpha \cos \theta & 0 & 0 \\
\cos \alpha \sin \theta & \cos \theta & -\sin \alpha \sin \theta & 0 & 0 \\
\sin \alpha & 0 & \cos \alpha & 0 & 0 \\
0 & 0 & 0 & 1 & 0 \\
0 & 0 & 0 & 0 & 1
\end{array}\right)
$$


and where

$$
\frac{\partial \Phi}{\partial \boldsymbol{\Phi}_{\mathrm{ss}}}=\left(\begin{array}{ccccc}
\frac{\rho u_{1}}{\|\mathbf{u}\|} & \frac{-\rho u_{2}}{\sqrt{u_{1}^{2}+u_{2}^{2}}} & \frac{-\rho u_{1} u_{3}}{\|\mathbf{u}\| \sqrt{u_{1}^{2}+u_{2}^{2}}} & \frac{\rho u_{1}}{c} & -\frac{u_{1}}{c^{2}} \\
\frac{\rho u_{2}}{\|\mathbf{u}\|} & \frac{\rho u_{1}}{\sqrt{u_{1}^{2}+u_{2}^{2}}} & \frac{-\rho u_{2} u_{3}}{\|\mathbf{u}\| \sqrt{u_{1}^{2}+u_{2}^{2}}} & \frac{\rho u_{2}}{c} & -\frac{u_{2}}{c^{2}} \\
\frac{\rho u_{3}}{\|\mathbf{u}\|} & 0 & \frac{\rho \sqrt{u_{1}^{2}+u_{2}^{2}}}{\|\mathbf{u}\|} & \frac{\rho u_{3}}{c} & -\frac{u_{3}}{c^{2}} \\
0 & 0 & 0 & \frac{\rho}{c} & -\frac{1}{c^{2}} \\
\rho\|\mathbf{u}\| & 0 & 0 & \rho c\left(\frac{c v}{R}+\frac{M^{2}}{2}\right) & -\frac{1}{2} M^{2}
\end{array}\right)
$$

and its inverse is

$$
\frac{\partial \Phi_{\mathrm{ss}}}{\partial \boldsymbol{\Phi}}=\left(\begin{array}{ccccc}
\frac{u_{1}}{\rho\|\mathbf{u}\|} & \frac{u_{2}}{\rho\|\mathbf{u}\|} & \frac{u_{3}}{\rho\|\mathbf{u}\|} & \frac{-\|\mathbf{u}\|}{\rho} & 0 \\
\frac{-u_{2}}{\rho \sqrt{u_{1}^{2}+u_{2}^{2}}} & \frac{u_{1}}{\rho \sqrt{u_{1}^{2}+u_{2}^{2}}} & 0 & 0 & 0 \\
\frac{-u_{1} u_{3}}{\rho\|\mathbf{u}\| \sqrt{u_{1}^{2}+u_{2}^{2}}} & \frac{-u_{2} u_{3}}{\rho\|\mathbf{u}\| \sqrt{u_{1}^{2}+u_{2}^{2}}} & \frac{\sqrt{u_{1}^{2}+u_{2}^{2}}}{\rho\|\mathbf{u}\|} & 0 & 0 \\
\frac{-R}{c_{v}} \frac{u_{1}}{\rho c} & \frac{-R}{c_{v}} \frac{u_{2}}{\rho c} & \frac{-R}{c_{v}} \frac{u_{3}}{\rho c} & \frac{1}{2} \frac{R}{c_{v}} \frac{\|\mathbf{u}\|^{2}}{\rho c} & \frac{R}{c_{v}} \frac{1}{\rho c} \\
-\frac{R}{c_{v}} u_{1} & -\frac{R}{c_{v}} u_{2} & -\frac{R}{c_{v}} u_{3} & \frac{1}{2} \frac{R}{c_{v}}\|\mathbf{u}\|^{2}-c^{2} & \frac{R}{c_{v}}
\end{array}\right)
$$

As $\frac{\partial}{\partial x_{i}}=\frac{\partial \xi_{k}}{\partial x_{i}} \frac{\partial}{\partial \xi_{k}}$, let $\mathbf{A}_{\mathrm{ss}}^{i}=\frac{\partial \boldsymbol{\Phi}_{\mathrm{ss}}}{\partial \boldsymbol{\Phi}_{\mathrm{s}}} \mathbf{A}_{\mathrm{s}}^{k} \frac{\partial \boldsymbol{\Phi}_{\mathrm{s}}}{\partial \mathbf{\Phi}_{\mathrm{ss}}} \frac{\partial \xi_{i}}{\partial x_{k}}$, or equivalently $\mathbf{A}_{\mathrm{ss}}^{i}=\frac{\partial \boldsymbol{\Phi}_{\mathrm{ss}}}{\partial \mathbf{\Phi}^{k}} \mathbf{A}^{k} \frac{\partial \boldsymbol{\Phi}}{\partial \boldsymbol{\Phi}_{\mathrm{ss}}} \frac{\partial \xi_{i}}{\partial x_{k}}$, for $i=1, \ldots, 3$. Then:

$$
\mathbf{A}_{\mathrm{ss}}^{1}=\left(\begin{array}{ccccc}
q_{1} & 0 & 0 & c & 0 \\
0 & q_{1} & 0 & 0 & 0 \\
0 & 0 & q_{1} & 0 & 0 \\
c & 0 & 0 & q_{1} & 0 \\
0 & 0 & 0 & 0 & q_{1}
\end{array}\right), \quad \mathbf{A}_{\mathrm{ss}}^{2}=\left(\begin{array}{ccccc}
0 & 0 & 0 & 0 & 0 \\
0 & 0 & 0 & c & 0 \\
0 & 0 & 0 & 0 & 0 \\
0 & c & 0 & 0 & 0 \\
0 & 0 & 0 & 0 & 0
\end{array}\right), \quad \mathbf{A}_{\mathrm{ss}}^{3}=\left(\begin{array}{ccccc}
0 & 0 & 0 & 0 & 0 \\
0 & 0 & 0 & 0 & 0 \\
0 & 0 & 0 & c & 0 \\
0 & 0 & c & 0 & 0 \\
0 & 0 & 0 & 0 & 0
\end{array}\right) .
$$

which are very attractive to work with due to their sparseness and because $\mathbf{A}_{\mathrm{ss}}^{i}$ for $i=2,3$, are degenerated matrices. The Euler system of equations with symmetrizing variables and stream-wise coordinates is written as:

$$
\frac{\partial \boldsymbol{\Phi}_{\mathrm{ss}}}{\partial t}+\mathbf{A}_{\mathrm{ss}}^{i} \frac{\partial \boldsymbol{\Phi}_{\mathrm{ss}}}{\partial \xi_{i}}=0
$$

Systems (C.10), (4), and (B.5) are equivalent. 


\section{Appendix D. Primitive variables}

The primitive variables are

$$
\mathbf{\Phi}_{\mathrm{P}}=\left(u_{1} u_{2} u_{3} p T\right)^{\mathrm{T}}
$$

The Jacobians that are used to change from primitive to conservative variables are given as:

$$
\frac{\partial \Phi}{\partial \boldsymbol{\Phi}_{\mathrm{P}}}=\left(\begin{array}{ccccc}
\rho & 0 & 0 & \frac{U_{1}}{p} & -\frac{U_{1}}{T} \\
0 & \rho & 0 & \frac{U_{2}}{p} & -\frac{U_{2}}{T} \\
0 & 0 & \rho & \frac{U_{3}}{p} & -\frac{U_{3}}{T} \\
0 & 0 & 0 & \frac{\rho}{p} & -\frac{\rho}{T} \\
U_{1} & U_{2} & U_{3} & \frac{1}{R}\left(\frac{1}{2} \frac{\|\mathbf{u}\|^{2}}{T}+c_{v}\right) & -\frac{1}{2} \frac{\rho\|\mathbf{u}\|^{2}}{T}
\end{array}\right)
$$

and

$$
\frac{\partial \Phi_{\mathrm{P}}}{\partial \boldsymbol{\Phi}}=\left(\begin{array}{ccccc}
\frac{1}{\rho} & 0 & 0 & \frac{u_{1}}{\rho} & 0 \\
0 & \frac{1}{\rho} & 0 & \frac{u_{2}}{\rho} & 0 \\
0 & 0 & \frac{1}{\rho} & \frac{u_{3}}{\rho} & 0 \\
\frac{R}{c_{v}} u_{1} & \frac{R}{c_{v}} u_{2} & \frac{R}{c_{v}} u_{3} & \frac{1}{2} \frac{R}{c_{v}}\|\mathbf{u}\|^{2} & \frac{R}{c_{v}} \\
-\frac{u_{1}}{c_{v} \rho} & -\frac{u_{2}}{c_{v} \rho} & -\frac{u_{3}}{c_{v} \rho} & \frac{1}{c_{v} \rho}\left(\|\mathbf{u}\|^{2}-\frac{E}{\rho}\right) & \frac{1}{c_{v} \rho}
\end{array}\right)
$$

\section{Appendix E. Group velocity of a plane wave}

Appendix E.1. 2D case

Let us consider $\mathbf{n}=\left(n_{1}, n_{2}\right)=\|\mathbf{n}\|(\cos \theta, \sin \theta)$ in the two dimensional case, where $\theta=$ $\arctan \left(\frac{n_{2}}{n_{1}}\right)$ is the angle of the wave propagation direction, $\mathbf{n}$, relative to the $x_{1}$-axis. Then the wave speeds can be expressed as $\lambda_{i}(\theta(\mathbf{n}))$. Thus, the group velocities (Eq. (15)) can be expressed as

$$
\mathbf{g}_{i}(\mathbf{n})=\left(\begin{array}{cc}
\cos \theta & -\sin \theta \\
\sin \theta & \cos \theta
\end{array}\right)\left(\begin{array}{c}
\lambda_{i}(\theta) \\
\lambda_{i}^{\prime}(\theta)
\end{array}\right)
$$

where $\lambda_{i}^{\prime}(\theta)=\frac{\partial \lambda_{i}}{\partial \theta}$. 


\section{Appendix E.2. 3D case}

Extended to the three dimensional case, let $\mathbf{n}=\left(n_{1}, n_{2}, n_{3}\right)$ be the wave propagation direction and $\theta$ and $\alpha$ the wave propagation angles such that

$$
\left\{\begin{array} { r l } 
{ \operatorname { s i n } \theta } & { = \frac { n _ { 2 } } { \sqrt { n _ { 1 } ^ { 2 } + n _ { 2 } ^ { 2 } } } } \\
{ \operatorname { c o s } \theta } & { = \frac { n _ { 1 } } { \sqrt { n _ { 1 } ^ { 2 } + n _ { 2 } ^ { 2 } } } } \\
{ \theta } & { = \operatorname { a r c t a n } ( \frac { n _ { 2 } } { n _ { 1 } } ) }
\end{array} \quad \left\{\begin{array}{rl}
\sin \alpha & =\frac{n_{3}}{\|\mathbf{n}\|} \\
\cos \alpha & =\frac{\sqrt{n_{1}^{2}+n_{2}^{2}}}{\|\mathbf{n}\|} \\
\alpha & =\arctan \left(\frac{n_{3}}{\sqrt{n_{1}^{2}+n_{2}^{2}}}\right),
\end{array}\right.\right.
$$

or equivalently,

$$
\left\{\begin{array}{l}
n_{1}=\|\mathbf{n}\| \cos \alpha \cos \theta \\
n_{2}=\|\mathbf{n}\| \cos \alpha \sin \theta \\
n_{3}=\|\mathbf{n}\| \sin \alpha
\end{array}\right.
$$

Then the group velocities (Eq. (15)) can be computed as

$$
\mathbf{g}_{i}(\mathbf{n})=\left(\begin{array}{ccc}
\cos \theta \cos \alpha & -\frac{\sin \theta}{\cos \alpha} & -\cos \theta \sin \alpha \\
\sin \theta \cos \alpha & \frac{\cos \theta}{\cos \alpha} & -\sin \theta \sin \alpha \\
\sin \alpha & 0 & \cos \alpha
\end{array}\right)\left(\begin{array}{c}
\lambda_{i}(\theta, \alpha) \\
\frac{\partial \lambda_{i}}{\partial \theta} \\
\frac{\partial \lambda_{i}}{\partial \alpha}
\end{array}\right) .
$$

\section{Appendix F. VLR in the conservative variables}

The VLR preconditioner in the conservative set of variables:

$$
\mathbf{P}_{\mathrm{VLR}}=\left(\begin{array}{lllll}
a u_{1} u_{1}+\tau & a u_{1} u_{2} & a u_{1} u_{3} & b u_{1} & c u_{1} \\
a u_{1} u_{2} & a u_{2} u_{2}+\tau & a u_{2} u_{3} & b u_{2} & c u_{2} \\
a u_{3} u_{1} & a u_{3} u_{2} & a u_{3} u_{3}+\tau & b u_{3} & c u_{3} \\
d u_{1} & d u_{2} & d u_{3} & e & f \\
g u_{1} & g u_{2} & g u_{3} & h & i
\end{array}\right),
$$


where

$$
\begin{aligned}
a & =\frac{1}{\|\mathbf{u}\|^{2}}\left(1+\frac{\tau}{\beta^{2}}-\tau\right)+\frac{1}{c^{2}}\left(\frac{R}{c_{v}} \frac{\tau\left(1-M^{2}\right)}{\beta^{2}}-\frac{\tau}{\beta^{2}}+\frac{R}{c_{v}}\right) \\
b & =\frac{\tau\left(M^{2}-1\right)}{\beta^{2}}\left(1+\frac{1}{2} \frac{R}{c_{v}} M^{2}\right)-\frac{1}{2} \frac{R}{c_{v}} M^{2} \\
c & =\frac{R}{c_{v}} \frac{1}{c^{2}}\left(\frac{\tau\left(M^{2}-1\right)}{\beta^{2}}-1\right) \\
d & =\frac{R}{c_{v}} \frac{1}{c^{2}}\left(1-\frac{\tau M^{2}}{\beta^{2}}\right)-\frac{\tau}{c^{2} \beta^{2}} \\
e & =1+\frac{\tau M^{2}}{\beta^{2}}+\frac{1}{2} \frac{R}{c_{v}} M^{2}\left(\frac{\tau M^{2}}{\beta^{2}}-1\right) \\
f & =\frac{R}{c_{v}} \frac{1}{c^{2}}\left(\frac{\tau M^{2}}{\beta^{2}}-1\right) \\
g & =1+\left(1-\frac{c_{v}}{R}\right) \frac{\tau}{\beta^{2}}+\left(\frac{R}{c_{v}}-\frac{3}{2}\right) \frac{\tau M^{2}}{\beta^{2}}+\frac{1}{2} \frac{R}{c_{v}} M^{2}\left(1-\frac{\tau M^{2}}{\beta^{2}}\right), \\
h & =\|\mathbf{u}\|^{2}\left[\left(\frac{c_{v}}{R}-1+M^{2}\left(1-\frac{1}{2} \frac{R}{c_{v}}\right)\right) \frac{\tau}{\beta^{2}}+\frac{1}{4} \frac{R}{c_{v}} M^{2}\left(\frac{\tau M^{2}}{\beta^{2}}-1\right)-\frac{1}{2}\right], \\
i & =\left(1-\frac{R}{c_{v}}\right) \frac{\tau M^{2}}{\beta^{2}}+\frac{1}{2} \frac{R}{c_{v}} M^{2}\left(\frac{\tau M^{2}}{\beta^{2}}-1\right) .
\end{aligned}
$$

\section{Appendix G. CM in the conservative variables}

The CM preconditioner in the conservative set of variables:

$$
\mathbf{P}_{\mathrm{CM}}=\left(\begin{array}{ccccc}
1+\frac{u_{1} u_{1}}{c_{p} T} & \frac{u_{1} u_{2}}{c_{p} T} & \frac{u_{1} u_{3}}{c_{p} T} & \frac{u_{1}}{c_{p} T} a & -\frac{u_{1}}{c_{p} T} \\
\frac{u_{2} u_{1}}{c_{p} T} & 1+\frac{u_{2} u_{2}}{c_{p} T} & \frac{u_{2} u_{3}}{c_{p} T} & \frac{u_{2}}{c_{p} T} a & -\frac{u_{2}}{c_{p} T} \\
\frac{u_{3} u_{1}}{c_{p} T} & \frac{u_{3} u_{2}}{c_{p} T} & 1+\frac{u_{3} u_{3}}{c_{p} T} & \frac{u_{3}}{c_{p} T} a & -\frac{u_{3}}{c_{p} T} \\
\frac{u_{1}}{c_{p} T} & \frac{u_{2}}{c_{p} T} & \frac{u_{3}}{c_{p} T} & 1+\frac{1}{c_{p} T} a & -\frac{1}{c_{p} T} \\
u_{1} b & u_{2} b & u_{3} b & a b & -\frac{1}{2} \frac{\|\mathbf{u}\|^{2}}{c_{p} T}
\end{array}\right),
$$

where $a=\frac{c_{v}}{R} \beta M_{r}^{2}-\frac{1}{2}\|\mathbf{u}\|^{2}$ and $b=1+\frac{1}{2} \frac{\|\mathbf{u}\|^{2}}{c_{p} T}$.

\section{Appendix H. CM in the symmetrizing variables and streamline coordinates}

The CM preconditioner in the symmetrizing variables and streamline coordinates:

$$
\mathbf{P}_{\mathrm{CM}}^{\mathrm{ss}}=\left(\begin{array}{ccccc}
1 & 0 & 0 & 0 & 0 \\
0 & 1 & 0 & 0 & 0 \\
0 & 0 & 1 & 0 & 0 \\
0 & 0 & 0 & M_{r}^{2} & -\frac{M_{r}^{2}}{\rho c} \\
0 & 0 & 0 & 0 & 1
\end{array}\right)
$$


[1] B. van Leer, W. Lee, P. Roe, Characteristic time-stepping or local preconditioning of the Euler equations, AIAA Paper 91-1552.

[2] Y.-H. Choi, C. Merkle, The application of preconditioning in viscous flows, Journal of Computational Physics 105 (2) (1993) 207 - 223. doi:http://dx.doi.org/10.1006/jcph.1993.1069.

URL http://www.sciencedirect.com/science/article/pii/S0021999183710697

[3] C. L. Merkle, M. Athavale, Time-accurate unsteady incompressible flow algorithms based on artificial compressibility, AIAA Paper 87-1137.

[4] A. Jameson, Time dependent calculations using multigrid with application to unsteady flows past airfoils and wings, AIAA Paper 91-1596.

[5] E. Turkel, V. N. Vatsa, Local preconditioners for steady and unsteady flow applications, ESAIM: Mathematical Modelling and Numerical Analysis 39 (2005) 515-535. doi:10.1051/m2an:2005021.

URL http://www.esaim-m2an.org/article_S0764583X0500021X

[6] A. Chorin, A numerical method for solving incompressible viscous flow problems, Journal of Computational Physics 2 (1) (1967) 12-26.

[7] E. Turkel, Preconditioned methods for solving the incompressible and low speed compressible equations, Journal of Computational Physics 72 (1987) 277-298.

[8] W. T. Lee, Local preconditioning of the Euler equations, Ph.D. thesis, University of Michigan (1991).

[9] D. Lee, The Design of Local Navier-Stokes Preconditioning for Compressible Flow, Journal of Computational Physics 144 (2) (1998) 460 - 483. doi:http://dx.doi.org/10.1006/jcph.1998.5994.

URL http://www.sciencedirect.com/science/article/pii/S0021999198959940

[10] D. Lee, Local preconditioning of the Euler and Navier-Stokes equations, Ph.D. thesis, University of Michigan (1996).

[11] N. Nigro, M. Storti, S. Idelsohn, T. Tezduyar, Physics based GMRES preconditioner for compressible and incompressible Navier-Stokes equations, Computer Methods in Applied Mechanics and Engineering 154 (3-4) (1998) 203-228. doi:http://dx.doi.org/10.1016/S0045-7825(97)00129-1.

URL http://www.sciencedirect.com/science/article/pii/S0045782597001291

[12] J. M. Weiss, W. A. Smith, Preconditioning applied to variable and constant density flows, AIAA Journal 33 (11) (1995) 2050-2057.

[13] W. Briley, L. Taylor, D. Whitfield, High-resolution viscous flow simulations at arbitrary Mach number, Journal of Computational Physics 184 (1) (2003) 79 - 105. doi:http://dx.doi.org/10.1016/S0021-9991(02)00018-9.

URL http://www.sciencedirect.com/science/article/pii/S0021999102000189

[14] D. L. Darmofal, B. van Leer, Local preconditioning of the Euler equations: A characteristic interpretation, Von Karman Institute Lecture Series 1999-03.

[15] A. G. Godfrey, R. W. Walters, B. van Leer, Preconditioning for the Navier-Stokes equations with finite-rate chemistry, AIAA Paper 93-0535.

[16] E. Turkel, Review of preconditioning methods for fluid dynamics, Applied Numerical Mathematics 12 (13) (1993) 257 - 284, Special Issue. doi:http://dx.doi.org/10.1016/0168-9274(93)90122-8.

URL http://www.sciencedirect.com/science/article/pii/0168927493901228 
[17] E. J. López, N. M. Nigro, S. S. Sarraf, S. M. Damián, Stabilized finite element method based on local preconditioning for unsteady compressible flows in deformable domains with emphasis on the low Mach number limit application, International Journal for Numerical Methods in Fluids 69 (1) (2012) 124-145. doi:10.1002/fld.2547. URL http://dx.doi.org/10.1002/fld.2547

[18] M. Moragues, G. Bernardino, M. Vzquez, G. Houzeaux, Fourier stability analysis and local Courant number of the preconditioned variational multiscale stabilization (P-VMS) for Euler compressible flow, Computer Methods in Applied Mechanics and Engineering 301 (2016) 28 - 51. doi:http://dx.doi.org/10.1016/j.cma.2015.12.008.

URL http://www.sciencedirect.com/science/article/pii/S0045782515004107

[19] M. Moragues Ginard, Variational multiscale stabilization and local preconditioning for compressible flow, Ph.D. thesis, Universitat Politècnica de Catalunya, Barcelona, Spain (2016).

[20] M. Vázquez, M. Pérez, M. Moragues, G. Houzeaux, Parallel monolithic implicit solver for compressible flows, in: Proceedings: PANACM Conference, Buenos Aires, Argentina, 2015.

[21] R. Courant, K. Friedrichs, H. Lewy, On the partial difference equations of mathematical physics, IBM Journal Res. Dev. 11 (2) (1967) 215-234.

[22] D. L. Darmofal, B. van Leer, Local Preconditioning: Manipulating Mother Nature to Fool Father Time, in: D. A. Caughey, M. M. Hafez (Eds.), Computing the Future II: Frontiers of Computational Fluid Dynamics 1998, World Scientific Publishing Co. Pte. Ltd., Singapore, 1998, pp. $211-240$.

[23] J. F. Lynn, Multigrid solution of the Euler equations with local preconditioning, Ph.D. thesis, University of Michigan (1995).

[24] D. Darmofal, P. Schmid, The importance of eigenvectors for local preconditioners of the Euler equations, Journal of Computational Physics 127 (2) (1996) 346 - 362. doi:http://dx.doi.org/10.1006/jcph.1996.0180.

URL http://www.sciencedirect.com/science/article/pii/S0021999196901801

[25] D. Lee, Design Criteria for Local Euler Preconditioning, Journal of Computational Physics 144 (2) (1998) 423 - 459. doi:http://dx.doi.org/10.1006/jcph.1998.5993.

URL http://www.sciencedirect.com/science/article/pii/S0021999198959939

[26] D. Lee, B. van Leer, Progress in local preconditioning of the Euler and Navier-Stokes equations, AIAA Paper 93-3328-CP.

[27] D. Lee, B. van Leer, J. F. Lynn, A local Navier-Stokes preconditioner for all Mach and cell Reynolds number, AIAA Paper 97-2024.

[28] A. Quarteroni, A. Valli, Numerical Approximation of Partial Differential Equations, Springer, 1994.

[29] T. J. R. Hughes, T. Tezduyar, Finite element methods for first-order hyperbolic systems with particular emphasis on the compressible Euler equations, Comput. Methods Appl. Mech. Engrg. 45 (1984) 217-284.

[30] T. J. R. Hughes, M. Mallet, A new finite element formulation for computational fluid dynamics: III. The generalized streamline operator for multidimensional advective-diffusive systems, Comp. Methods Appl. Mech. Engrg. 58 (1986) 305-328.

[31] G. J. Le Beau, S. E. Ray, S. K. Aliabadi, T. E. Tezduyar, SUPG finite element computation of compressible flows with the entropy and conservation variables formulations, Comp. Methods Appl. Mech. Engrg. 104 (1993) $397-422$. 
[32] T. E. Tezduyar, M. Senga, Stabilization and shock-capturing parameters in SUPG formulation of compressible flows, Computer Methods in Applied Mechanics and Engineering 195 (13-16) (2006) 1621 - 1632, a Tribute to Thomas J.R. Hughes on the Occasion of his 60th Birthday. doi:http://dx.doi.org/10.1016/j.cma.2005.05.032. URL http://www.sciencedirect.com/science/article/pii/S0045782505002999

[33] F. Shakib, T. J. R. Hughes, Z. Johan, A new finite element formulation for computational fluid dynamics: X. The compressible Euler and Navier-Stokes equations, Comput. Methods Appl. Mech. Engrg. 89 (1991) 141-291.

[34] T. Hughes, G. Scovazzi, T. E. Tezduyar, Stabilized methods for compressible flows, J. Sci. Comput. 43 (2010) $343-368$

[35] T. Hughes, Multiscale phenomena: Green's functions, the Dirichlet-to-Neumann formulation, subgrid scale models, bubbles and the origins of stabilized methods, Comput. Methods Appl. Mech. and Engrg. 127 (1995) $387-401$.

[36] T. J. R. Hughes, G. Feijoo, L. Mazzei, J. Quincy, The variational multiscale method - A paradigm for computational mechanics, Comput. Methods Appl. Mech. Engrg. 166 (1998) 3-24.

[37] F. Rispoli, R. Saavedra, A stabilized finite element method based on SGS models for compressible flows, Comput. Methods Appl. Mech. and Engrg. 196 (2006) 652-664.

[38] F. Rispoli, R. Saavedra, A. Corsini, T. E. Tezduyar, Computation of inviscid compressible flow with V-SGS stabilization and $Y Z \beta$ shock capturing, Int. J. Numer. Methods Fluids 54 (2007) 695-706.

[39] S. Marras, M. Moragues, M. Vázquez, O. Jorba, G. Houzeaux, A variational multiscale stabilized finite element method for the solution of the Euler equations of nonhydrostatic stratified flows, Journal of Computational Physics 236 (2013) 380 - 407. doi:http://dx.doi.org/10.1016/j.jcp.2013.06.006.

[40] S. Marras, M. Moragues, M. Vázquez, O. Jorba, G. Houzeaux, Simulations of moist convection by a variational multiscale stabilized finite element method, Journal of Computational Physics 252 (2013) 195 - 218. doi:http://dx.doi.org/10.1016/j.jcp.2013.06.006.

[41] S. Marras, J. F. Kelly, M. Moragues, A. Müller, M. A. Kopera, M. Vázquez, F. X. Giraldo, G. Houzeaux, O. Jorba, A review of element-based Galerkin methods for numerical weather prediction: Finite elements, spectral elements, and discontinuous Galerkin, Archives of Computational Methods in Engineering (2015) 150doi:10.1007/s11831-015-9152-1.

URL http://dx.doi.org/10.1007/s11831-015-9152-1

[42] S. S. Collis, Y. Chang, The DG/VMS method for unified turbulence simulation, AIAA paper 3124 (2002) $24-27$.

[43] B. Koobus, F. Charbel, A variational multiscale method for the large eddy simulation of compressible turbulent flows on unstructured meshes - application to vortex shedding, Comp. Methods Appl. Mech. Engr. 193 (2004) $1367-1383$.

[44] C. Farhat, A. K. Rajasekharan, B. Koobus, A dynamic variational multiscale method for large eddy simulations on unstructured meshes, Computer Methods in Applied Mechanics and Engineering 195 (1316) (2006) 1667 - 1691, a Tribute to Thomas J.R. Hughes on the Occasion of his 60th Birthday. doi:http://dx.doi.org/10.1016/j.cma.2005.05.045.

URL http://www.sciencedirect.com/science/article/pii/S0045782505003014

[45] H. Ouvrard, B. Koobus, A. Dervieux, M. V. Salvetti, Classical and variational multiscale LES of the 
flow around a circular cylinder on unstructured grids, Computers \& Fluids 39 (7) (2010) 1083 - 1094. doi:http://dx.doi.org/10.1016/j.compfluid.2010.01.017.

URL http://www.sciencedirect.com/science/article/pii/S0045793010000319

[46] V. Levasseur, P. Sagaut, F. Chalot, A. Davroux, An entropy-variable-based VMS/GLS method for the simulation of compressible flows on unstructured grids, Computer Methods in Applied Mechanics and Engineering 195 (912) (2006) 1154 - 1179. doi:http://dx.doi.org/10.1016/j.cma.2005.04.009.

URL http://www.sciencedirect.com/science/article/pii/S0045782505001568

[47] F. van der Bos, J. J. van der Vegt, B. J. Geurts, A multiscale formulation for compressible turbulent flow suitable for general variational discretization techniques, Comput. Methods Appl. Mech. Engrg. 196 (2007) 2863-2875.

[48] R. Codina, J. Blasco, Analysis of a stabilized finite element approximation of the transient convection-diffusionreaction equation using orthogonal subscales, Comput. Visusl. Sci. 4 (2002) 167-174.

[49] R. Codina, Stabilized finite element approximation of transient incompressible flows using orthogonal subscales, Comput. Methods Appl. Mech. Engrg. 191 (2002) 4295-4321.

[50] G. Hauke, Simple stabilizing matrices for the computation of compressible flows in primitive variables, Computer Methods in Applied Mechanics and Engineering 190 (51-52) (2001) 6881 - 6893. doi:http://dx.doi.org/10.1016/S0045-7825(01)00267-5.

URL http://www.sciencedirect.com/science/article/pii/S0045782501002675

[51] T. R. Hughes, The finite element method: Linear static and dynamic finite element analysis, 2nd Edition, Dover Publications Inc., 2000.

[52] O. Arias, O. Falcinelli, N. Fico, S. Elaskar, Finite volume simulation of a flow over a NACA 0012, Mecánica Computacional, Asociación Argentina de Mecánica Computacional 26 (2007) 3097-3116.

[53] V. Schmitt, F. Charpin, Pressure Distributions on the ONERA-M6-Wing at Transonic Mach Numbers, AGARD Advisory Report No. 138, Experimental data base for computer program assessment by Fluid Dynamics Panel, Working Group 04.

[54] H. Luo, J. D. Baum, R. Lhner, A fast, matrix-free implicit method for compressible flows on unstructured grids, in: C.-H. Bruneau (Ed.), Sixteenth International Conference on Numerical Methods in Fluid Dynamics, Vol. 515 of Lecture Notes in Physics, Springer Berlin Heidelberg, 1998, pp. 73-78. doi:10.1007/BFb0106564.

URL http://dx.doi.org/10.1007/BFb0106564

[55] E. Turkel, Symmetrization of the fluid dynamic matrices with applications, Mathematics of Computation 27 (124) (1973) 729-736. 